\title{
Real Time Process Algebra with Infinitesimals
}

\author{
J.C.M. Baeten \\ Department of Computer Science, Eindhoven University of Technology, \\ Eindhoven, The Netherlands
}

J.A. Bergstra

Programming Research Group, University of Amsterdam, Amsterdam, The Netherlands, and Department of Philosophy, Utrecht University, Utrecht, The Netherlands

\begin{abstract}
We consider a model of the real time process algebra of $[1,2,3]$ based on the nonstandard reals. As a subalgebra, we obtain a theory in which the urgent actions of ATP, TiCCS, TeCCS can be modeled.

Note: This research was supported in part by ESPRIT basic research action 7166, CONCUR2. The second author also is partially supported by ESPRIT basic research action 6454, CONFER.
\end{abstract}

\section{Introduction.}

An important motivation for our work on timed process algebra $([1,2,3])$ is that we intend to present a uniform framework, in which all constructs that occur in the literature on timed process algebra can be expressed. In [1], we present our real time absolute time process algebra, in [3] we achieve a unified treatment of absolute time and relative time expressions. In [2], we define many discrete time constructs from the literature, both absolute and relative time ones, in our real time process algebra, and define suitable discrete time subalgebras. This paper is concerned with immediate or urgent actions, as they appear in TiCCS [11,12], ATP [13], TeCCS [16], TiCCS [7].

As an example, consider the TiCCS expression (2).a.b.0. Intuitively, this expression denotes the process that, after an initial delay of 2 time units, executes first $a$ and then $b$ instantaneously, and then terminates. Now, in our real time process algebra $\mathrm{ACP} \rho$, two actions cannot be executed consecutively at the same point in time, so the process $a(2) \cdot b(2)$ will deadlock after executing the action $a$ at time 2 , and $b$ will not be executed. Nevertheless, we can define the process above in an extension of ACP $\rho$. To this end, we extend our time domain to include infinitesimals, and model the TiCCS expression above by a process that first executes $a$ and then $b$ at time points both infinitely close to 2 , with the difference of execution times a positive infinitesimal.

This paper describes the extension of ACP $\rho$ to a more general time domain, describes the relation of $\mathrm{ACP} \rho$ over the (standard) positive reals with ACP $\rho$ over a time 
domain including nonstandard reals, and defines suitable subalgebras including urgent actions. Some of these subalgebras correspond to timed process algebras found in the literature.

We give an overview of the systems that we will consider. We start with the system BPA $\sqrt{ } \mathrm{I}$, real time process algebra with initial abstraction and integration. By adding parallel composition and communication, we obtain ACP $\sqrt{ } \mathrm{I}$. In this system (over a time domain involving nonstandard reals) we can define urgent actions. We can also directly axiomatise algebras based on urgent actions instead of our usual real time actions. In this way, we obtain the absolute time algebras BPAs $\rho$, PAs $\rho$, ACPs $\rho$, and the relative time algebras BPAst, BPA $\delta$ st, PAst, ACPst. By adding initial abstraction to

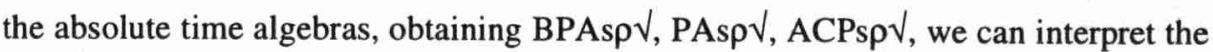
relative time ones in them. Figure 1 gives an overview of all algebras we consider, and the embedding relations between them. Boxes with rounded corners denote relative time algebras, square boxes denote absolute time algebras.

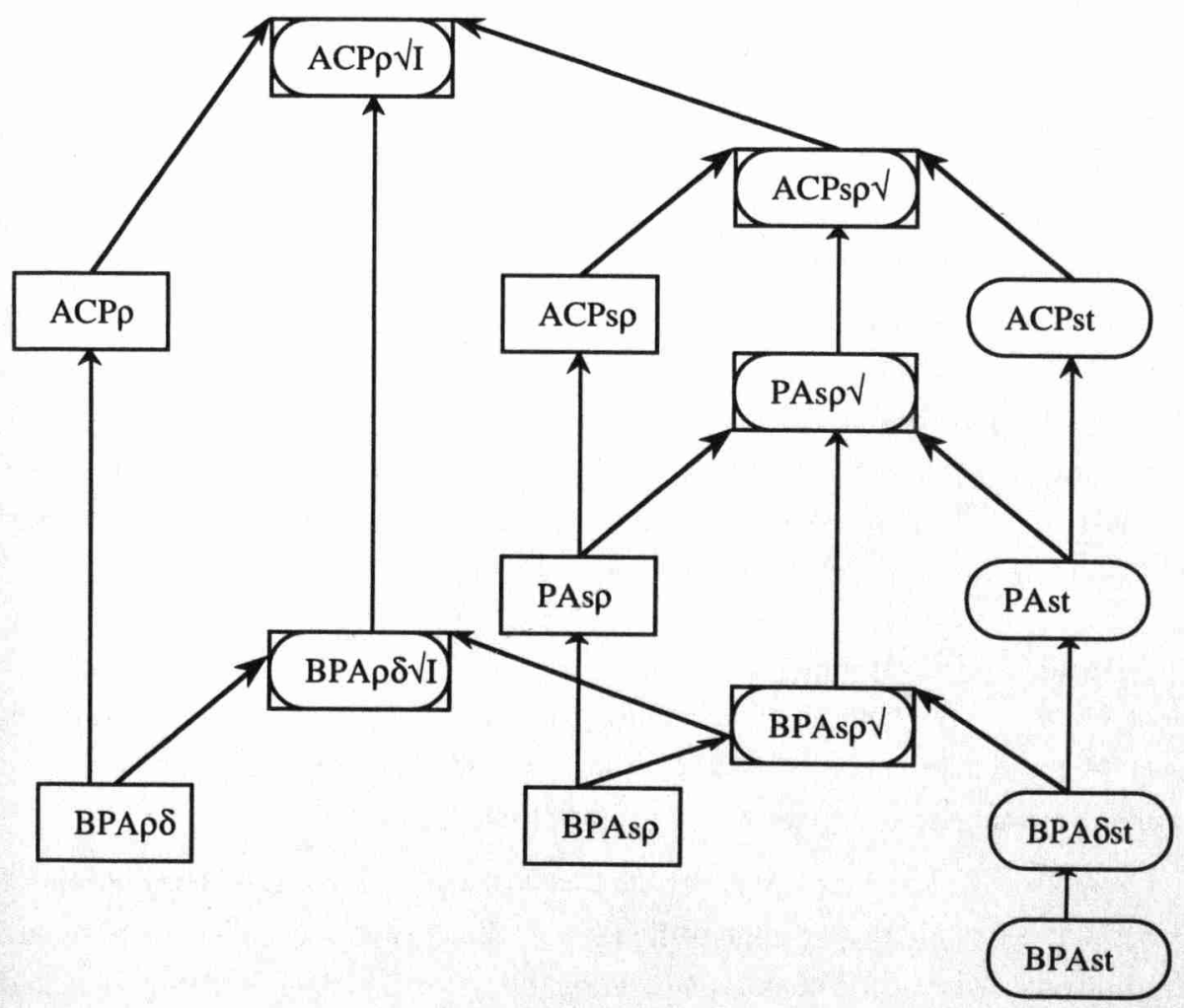

FIGURE 1. 
ACKNOWLEDGEMENT. We thank Steven Klusener (CWI Amsterdam) for his useful comments and suggestions, and Peter van Emde Boas (University of Amsterdam) for his suggestion to use nonstandard reals or surreal numbers.

\section{Real time process algebra with initial abstraction operator.}

We will first consider the axiom system ACP $\sqrt{ }$ I. It describes the real time process algebra of [1] as presented in [2]. The reader is assumed to be familiar with [2], as we only indicate the differences with that paper. For ACP $\rho \sqrt{I}$, we have a bisimulation model $M_{A}^{*}$ as outlined in [2].

Having available $M_{A}^{*}$, the various operators and constants of urgent time process algebra are defined in it. Then, as a subject, nonstandard real time process algebra reduces to an investigation of certain reducts and subalgebras of $\mathrm{M}_{\mathrm{A}}^{*}$ as well as their axiomatic description.

\subsection{Time domain.}

We assume that we have a time domain $\mathcal{T}$. We have the following requirements on $\mathcal{T}$ :

i. Tis linearly ordered by $<$

ii. 0 is the smallest element of $\mathcal{T}$

iii. $\mathcal{T}$ is closed under addition + .

In [1], we used the supremum of a subset of $\mathcal{T}$ in de definition of the ultimate delay operator, so we needed completeness of $\mathcal{T}$. We reformulate this operator in this paper (as we did in [BAB93]) so that this requirement is not needed anymore. Examples of time domains satisfying these requirements are $\mathbb{N}$, the naturals, $\mathbb{Q} \geq 0$, the non-negative rationals, $\mathbb{R}_{\geq 0}$, the non-negative reals, or $* \mathbb{R}_{\geq 0}$, the non-standard non-negative reals. Our prefered time domain, the one we used in $[1,2,3]$, is $\mathbb{R}_{\geq 0}$.

\subsection{Basic process algebra.}

$A$ is the set of (symbolic) atomic actions. The set $A$ is a parameter of the theory. $\delta$ denotes inaction. We put $A_{\delta}=A \cup\{\delta\}$. The set of constants is the set of atomic actions with time parameter, $A T$, given by $A T=\left\{a(t) \mid a \in A_{\delta}, t \in \mathcal{T}\right\}$.

Real Time Basic Process Algebra with Deadlock (BPApס) has two binary operators ,$+ \therefore \mathrm{P} \times \mathrm{P} \rightarrow \mathrm{P}$; alternative composition (weak choice) and sequential composition,

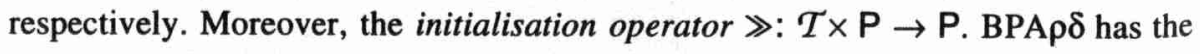
axioms given in $[1,2]$. 


\subsection{Algebra of Communicating Processes.}

In order to formulate communication between processes, we assume we have given a communication function I on $A_{\delta}$. An axiomatization of parallel composition with communication uses the left merge operator $\mathbb{L}$, the communication merge operator $I$, and the encapsulation operator $\partial_{\mathrm{H}}$ of [6]. Moreover, an extra auxiliary operator is needed. In $[1,2,3]$ we used the ultimate delay operator and the bounded initialization operator as auxiliary operators. However, in order to define the ultimate delay of an integral expression, we need the supremum of a subset of $\mathcal{T}$. In the time domain $\mathbb{R}_{\geq 0}$, such a supremum will always exist (or is infinite), but later on, we will consider a time domain where suprema need not exist. For this reason, we will use a different auxiliary operator here. Since the axiomatisation of merge used before only needed expressions of the form $X \gg U(Y)$ (with $U$ the ultimate delay and $\gg$ the bounded initialisation), we will have a new binary operator (left strong choice) for this combination. It turns out that this operator is exactly one half of the strong choice operator $\oplus$ of ATP, and that explains the name.

Thus, we have the operator $\oplus: P \times P \rightarrow P$ (left strong choice) and $X \oplus Y$ denotes intuitively that part of $X$, that can start before an initial action of $Y$. Notice the similarity of the axioms for the left strong choice operator with the axioms of the unless operator from $[4,5]$. The latter binary operator also describes a filtering of the first argument by the second. We call the present version of $\mathrm{ACP} \rho \operatorname{ACP} \rho(\boxplus)$. In table 1 , we show the axioms that do not appear in [2], that replace the axioms using ultimate delay or bounded initialisation. The axioms for left merge have a condition because they do not hold for processes involving relative time notation. We have $a, b \in A_{\delta}$.

\begin{tabular}{|c|c|}
\hline$t<s \Rightarrow a(t) \oplus b(s)=a(t)$ & LSC1 \\
\hline$t \geq s \Rightarrow a(t) \oplus b(s)=\delta(s)$ & LSC2 \\
\hline$X \oplus Y \cdot Z=X \oplus Y$ & LSC3 \\
\hline$X \oplus(Y+Z)=(X \oplus Y)+(X \oplus Z)$ & LSC4 \\
\hline$X \cdot Y \oplus Z=(X \oplus Z) \cdot Y$ & LSC5 \\
\hline$(X+Y) \oplus Z=(X \oplus Z)+(Y \oplus Z)$ & LSC6 \\
\hline$X=0 \gg Z \Rightarrow a(t) \mathbb{L} X=(a(t) \oplus X) \cdot X$ & $\mathrm{ATCM} 2 \oplus$ \\
\hline$Y=0 \gg Z \Rightarrow a(t) \cdot X \mathbb{L}=(a(t) \oplus Y) \cdot(X \| Y)$ & АТСM3円 \\
\hline
\end{tabular}

TABLE 1. New axioms of $\mathrm{ACP} \rho(\oplus)$.

\subsection{Relative time notation.}

We follow the approach to relative time of $[2,3]$. The basis for this approach to relative time notation is the initial abstraction operator. Let for $t \in \mathcal{T}, F(t)$ be a process in $P$, then 
$\sqrt{ } t . F(t)$ denotes a process that, when started at time $r$, proceeds as $F(r)$. In table 2, we show the new axioms for initial abstraction, that give the relation to the left strong choice operator.

\begin{tabular}{|ll|}
\hline$(\sqrt{t} . F) \oplus X=\sqrt{t} .(F \oplus t \gg X)$ & IA13 \\
$X \oplus(\sqrt{t} . F)=\sqrt{ } t .(t \gg X \oplus F)$ & IA14 \\
\hline
\end{tabular}

TABLE 2. Axioms for initial abstraction and left strong choice.

\subsection{Integration.}

We extend our real time process algebra by adding the integral operator, denoting a choice over a subset of $\mathcal{T}$. That is, if $V$ is a subset of $\mathcal{T}$, and $\mathrm{v}$ is a variable over $\mathcal{T}$, then $\int_{V \in V} P$ denotes the alternative composition of alternatives $P[t / v]$ for $t \in V$ (expression $P$ with $t \in \mathcal{T}$ substituted for variable $v$ ). In case the time domain is $\mathbb{R}_{\geq 0}$, this is an uncountable sum, and that explains the use of the notion of an integral. For more information, we refer the reader to [1] and [9].

In table $3, X$ is a process (that does not contain free variables), and $F, G$ are process expressions (that may contain free variables). We reformulate INT8: this only applies if the set $V$ has a supremum in $\mathcal{T}$. The theory presented thus far is called ACP $\rho \sqrt{ }$, ACP $\rho$ plus integration is ACPpI.

$$
\begin{aligned}
& \mathrm{t}=\sup \mathrm{V}, \mathrm{t} \notin V \Rightarrow \int_{\mathrm{V} \in \mathrm{V}} \delta(\mathrm{v})=\delta(\mathrm{t}) \\
& \int_{v \in V}(F \oplus X)=\left(\int_{v \in V} F\right) \oplus X \\
& \int_{v \in V}(X \oplus F)=X \oplus\left(\int_{v \in V} F\right)
\end{aligned}
$$

TABLE 3. Axioms for integration and left strong choice.

\subsection{Structured operational semantics.}

We obtain a real time transition system $T S(X)$ from a process expression $X$ as indicated in [BAB92]. Here, we just give the SOS rules for the left strong choice operator. We have $t \in \mathcal{T}, r \in \mathcal{T}\left\{\{0\}, x, x^{\prime}, y\right.$ are closed process expressions and $a \in A$. On these transition systems, we define bisimulation as usual. We extend with initial abstraction as in [2]. 


$$
\begin{aligned}
& \frac{\langle x, t\rangle \rightarrow\langle x, r\rangle,\langle y, t\rangle \rightarrow\langle y, r\rangle}{\langle x \oplus y, t\rangle \rightarrow\langle x \oplus y, r\rangle} \quad \frac{\langle x, t\rangle^{a(r)} \rightarrow\left\langle x^{\prime}, r\right\rangle,\langle y, t\rangle \rightarrow\langle y, r\rangle}{\langle x \oplus y, t\rangle^{a(r)}\left\langle\left\langle x^{\prime}, r\right\rangle\right.} \\
& \frac{\langle x, t\rangle \stackrel{a(r)}{\rightarrow}\langle\sqrt{ }, r\rangle,\langle y, t\rangle \rightarrow\langle y, r\rangle}{\left.\langle x \oplus y, t\rangle^{a(r)}\right)^{\rightarrow}\langle\sqrt{ }, r\rangle}
\end{aligned}
$$

TABLE 4. Structured operational semantics for left strong choice.

We present some extra axioms that are useful in the calculations to come, that hold in the model, and that can be derived from the theory for all closed process expressions. The numbering of these axioms corresponds to the numbering in [2].

\begin{tabular}{|ll|}
\hline$s \gg X \mathbb{} \gg \gg Y=(s \gg X \oplus r \gg Y) \mathbb{L} \gg r \gg Y$ & SI10 \\
$t \gg(X \oplus Y)=t \gg X \oplus t \gg Y$ & SI14 \\
$X+(X \oplus Y)=X$ & SI15 \\
$a(t) \oplus t \gg X=a(t)$ & SI16 \\
$t \gg X \oplus a(t)=\delta(t)$ & SI17 \\
\hline
\end{tabular}

TABLE 5. Standard initialisation axioms involving left strong choice.

\section{Nonstandard Reals.}

In this section, we consider two time domains: on the one hand we have the time domain $\mathbb{R} \geq 0$ of non-negative real numbers, on the other hand we consider a subdomain of $* \mathbb{R}$, the nonstandard model of the real numbers. For more information on nonstandard real numbers, see $[10,15]$. We use the following notations: $t \approx s$ means that $t, s$ are infinitely close together, $I=\{s \in * \mathbb{R} \mid s \approx 0, s>0\}$ is the set of positive infinitesimals and $t^{\circ} \in \mathbb{R}$ is the standard part of a finite $t \in * \mathbb{R}$, the standard real infinitely close to $t$. Note that the supremum over a bounded subset of $* \mathbb{R}$ need not exist.

As the extended time domain we use $\mathbb{R}_{\geq 0}+I=\mathbb{R}_{\geq 0} \cup\left\{p+\varepsilon: p \in \mathbb{R}_{\geq 0}, \varepsilon \in I\right\}$. Note that this set satisfies the requirements of section 2.1. Thus, this domain is linearly ordered, with 0 as least element, and is closed under addition. Also, it is closed under multiplication, and division by a positive standard real. Note that for $t \in \mathbb{R}_{\geq 0}+\mathrm{I}$, always $t^{\circ}$ exists and $t^{\circ} \leq t$.

\subsection{Definitions.}

We have the definitions in table 6 in the algebra over the extended time domain $\mathbb{R}_{\geq 0}+I$. We will always have $t, u \in \mathbb{R}_{\geq 0}+I, p, q \in \mathbb{R}_{\geq 0}, r \in \mathbb{R}_{>0}, \varepsilon, \eta \in I$. 


\begin{tabular}{|ll}
\hline$\tilde{a}[p]=\sqrt{ } t . \int_{\varepsilon \in I} a\left(p+t^{\circ}+\varepsilon\right)$ & $\tilde{\delta}[p]=\sqrt{ } t . \int_{\varepsilon \in I} \delta\left(p+t^{\circ}+\varepsilon\right)$ \\
$\widetilde{a}=\tilde{a}[0]$ & $\widetilde{\delta}=\tilde{\delta}[0]$ \\
$\tilde{a}(p)=\widetilde{\delta}+\int_{\varepsilon \in I} a(p+\varepsilon)$ & $\tilde{\delta}(p)=\widetilde{\delta}+\int_{\varepsilon \in I} \delta(p+\varepsilon)$ \\
$p \gg_{s} X=\widetilde{\delta}+p \gg X$ & $V_{s} p . F=\sqrt{ } t . F[t o / p]$ \\
$\sigma_{\approx}^{r}(X)=\sqrt{ } t .(t+r)^{\circ} \gg X$ & $r \gg_{s t} X=\sqrt{ } t .(t+r)^{\circ} \gg t^{\circ} \gg_{s} X$
\end{tabular}

TABLE 6. Standard time operators.

The standard absolute time signature, $\Sigma\left(\mathrm{ACP}_{\mathrm{s} \rho} \sqrt{ }\right)$, contains the following ingredients: $\quad \tilde{a}(p)\left(a \in A_{\delta}\right), \widetilde{\delta},+, \cdot$,

$$
\|, \mathbb{L}, \mid, \partial_{\mathrm{H}}(\mathrm{H} \subseteq \mathrm{A}), \gg_{\mathrm{s}}, \oplus,
$$

The standard relative time signature, $\Sigma\left(\mathrm{ACP}_{\mathrm{st}}\right)$, contains the following ingredients:

$$
\begin{aligned}
& \tilde{a}\left(a \in A_{\delta}\right),+, \cdot, \sigma_{\approx}^{r}, \\
& \|, \mathbb{L}, I, \partial_{H}(H \subseteq A), r \gg_{s t}, \oplus .
\end{aligned}
$$

Since we have $\tilde{a}[0]=\tilde{\tilde{a}}$ and $\tilde{a}[r]=\sigma \approx_{\approx}^{r}(\tilde{a})$ for all $r>0$, this signature also contains all constants of the form $\tilde{a}[p]$.

The standard time signature, $\Sigma(\mathrm{ST})$, is given by $\Sigma(\mathrm{ST})=\Sigma\left(\mathrm{ACP}_{\mathrm{s} \rho} \sqrt{ }\right) \cup \Sigma\left(\mathrm{ACP}_{\mathrm{st}}\right)$.

\section{Standardly initialized processes.}

In sections 5 and 6, we will give direct axiomatisations of various subalgebras of ACP $\sqrt{I}$, that are generated by signature elements of in section 3. Many of these axioms are not valid in the full algebra $M_{A}^{*}$ over $\mathbb{R}_{\geq 0}+I$. In this section we define the notion of a standardly initialised process. The set of standardly initialised processes is closed under all operators of the standard time signature, and all axioms to be presented in sections 6 and 7 will hold in this algebra. We prove these facts at length in this section, but omitting some easy proofs. We obtain a subalgebra $\operatorname{si}^{-} \mathrm{M}_{A}^{*}$ of $\mathrm{M}_{\mathrm{A}}^{*}$. Similarly, we have a subalgebra si- $M_{A}$ of $M_{A}$ (containing absolute time processes only) and a subalgebra si- $M_{A}^{r}$ of $M_{A}^{r}$ (containing relative time processes only).

\subsection{Definition.}

Let $X \in P^{\star}$. We say $X$ is a standardly initialised process, $X \in S I P$, if $X=\sqrt{S}_{s} p . p \gg_{s} X$. 
4.2 Theorem. The following statements are equivalent:
i. $X \in S I P$
ii. $X=\sqrt{t} \cdot t^{\circ} \gg_{s} X$
iii. $X=X+\widetilde{\delta} \wedge X=\sqrt{ } t . t^{\circ} \gg X$.

Proof: $\mathrm{i} \Leftrightarrow$ ii: By definition of $\sqrt{ }_{\mathbf{s}}$.

ii $\Leftrightarrow$ iii: If $X=\sqrt{ } t$. $t^{\circ} \gg_{s} X$, then

$$
\begin{aligned}
t \gg X & =t \gg \sqrt{ } u . u^{\circ} \gg_{s} X=t \gg\left(t^{\circ} \gg_{s} X+\widetilde{\delta}\right)=t \gg\left(t^{\circ} \gg_{s} X\right)+t \gg \widetilde{\delta}= \\
& =t \gg\left(\sqrt{ } u \cdot u^{\circ} \gg_{s} X\right)+t \gg \widetilde{\delta}=t \gg X+t \gg \widetilde{\delta}=t \gg(X+\widetilde{\delta}) .
\end{aligned}
$$

By extensionality (IA5) $X=X+\widetilde{\widetilde{\delta}}$.

Thus $X=\sqrt{t}$. $t^{\circ} \gg_{s} X \Rightarrow X=X+\widetilde{\delta}$, and $X=X+\widetilde{\delta}$ holds in both ii and iii. Using this,

$$
\begin{aligned}
& t \gg \sqrt{ } u . u^{\circ} \gg_{s} X=t \gg\left(t^{\circ} \gg X+\delta \tilde{\delta}\right)=t \gg\left(t^{\circ} \gg X\right)+t \gg \widetilde{\delta}= \\
& =t \gg t^{\circ} \gg X+t \gg t^{\circ} \gg \widetilde{\delta}=t \gg t^{\circ} \gg(X+\tilde{\delta})=t \gg t^{\circ} \gg X= \\
& =t \gg\left(\sqrt{ } u . u^{\circ} \gg X\right) \text {. Again by extensionality (IA5) } \sqrt{ } t . t^{\circ} \gg_{s} X=\sqrt{ } t . t^{\circ} \gg X \text {. }
\end{aligned}
$$

\subsection{Note.}

A simple example of a process that is not standardly initialised is $\sqrt{t}$. a(t+1). This process does satisfy $X=X+\widetilde{\delta}$. On the other hand, the process $\sqrt{t}$. $\int_{r=t+1} a(r)$ is standardly initialised. We will nevertheless see that the second process is not in the algebra generated by the signature $\Sigma(\mathrm{ST})$.

We proceed to show that the set of standardly initialised processes is closed under all operators of the standard time signature. Actually, we will show something stronger, viz. that both the condition $X=X+\widetilde{\delta}$ and the condition $X=\sqrt{ }$ t. $t^{\circ} \gg X$ are preserved by all operators of $\Sigma(S T)$. Thus, the algebra si-M $M_{A}^{*}$ is actually the intersection of two other interesting subalgebras of $M_{A}^{*}$. We first list some facts about initial abstraction that are proved as in [2].

4.4 Lemma. $\sqrt{ } t . F+\sqrt{ } t . G=\sqrt{ } t .(F+G), v_{t} . F=\sqrt{ } t . t \gg F, \sqrt{ } t . F \mid \sqrt{ } t . G=\sqrt{ } t .(F \mid G)$,

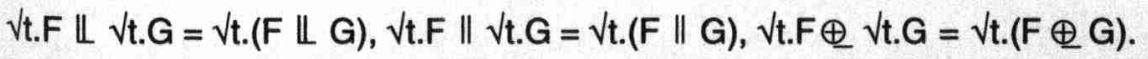

Proof: As in [2].

\subsection{Constants.}

We start to verify the two conditions of 4.2.iii by looking at the constants of $\Sigma$ (ST). 
4.5.1 Proposition: $\widetilde{\tilde{\delta}}+\widetilde{\delta}=\widetilde{\delta}$.

4.5.2 Proposition: $\widetilde{\widetilde{\delta}}=\sqrt{ } \mathbf{t} . \mathrm{t}^{\circ} \gg \widetilde{\delta}$.

Proof: This was already used in the proof of 4.2 . We give a more complete proof:

$\widetilde{\delta}=\sqrt{ }$ t. $\int_{\varepsilon \in I} \delta\left(t^{\circ}+\varepsilon\right)=\sqrt{ } t . \int_{\varepsilon \in I} t^{\circ} \gg \delta\left(t^{\circ}+\varepsilon\right)=\sqrt{ } t . t^{\circ} \gg \int_{\varepsilon \in I} \delta\left(t^{\circ}+\varepsilon\right)=\sqrt{ } t . t^{\circ} \gg \widetilde{\delta}$.

4.5.3 Proposition: $\tilde{a}(p)+\widetilde{\delta}=\tilde{a}(p)$.

4.5.5 Proposition: $\tilde{a}(p)=\sqrt{ } t . t^{\circ} \gg \tilde{a}(p)$.

$$
\begin{aligned}
& \text { Proof: } \tilde{a}(p)=\int_{\varepsilon \in I} a(p+\varepsilon)+\widetilde{\delta}=\int_{\varepsilon \in I} a(p+\varepsilon)+\sqrt{ } t . \int_{\varepsilon \in I} \delta\left(t^{\circ}+\varepsilon\right)= \\
& =\sqrt{ } t .\left(t \gg \int_{\varepsilon \in I} a(p+\varepsilon)+\int_{\varepsilon \in I} \delta\left(t^{\circ}+\varepsilon\right)\right)(I A 4) .
\end{aligned}
$$

Now we consider three cases:

i. if $t \leq p$, then $t \gg a(p+\varepsilon)=a(p+\varepsilon)=t^{\circ} \gg a(p+\varepsilon)$ for all $\varepsilon \in I$;

ii. if $t^{\circ}>p$, then $t \gg a(p+\varepsilon)=\delta(t)$ for all $\varepsilon \in I$, so $\left.t \gg \int_{\varepsilon \in I} a(p+\varepsilon)+\int_{\varepsilon \in I} \delta\left(t^{\circ}+\varepsilon\right)\right)=$

$$
\left.=\int_{\varepsilon \in \mathrm{I}} \delta\left(\mathrm{t}^{\circ}+\varepsilon\right)\right) \text { by ATA3; }
$$

iii. if $t=p+\varepsilon$ for some $\varepsilon \in I$, then $t^{\circ}=p$, so $\sqrt{t}$. $\left(t \gg \int_{\varepsilon \in I} a(p+\varepsilon)+\int_{\varepsilon \in I} \delta\left(t^{\circ}+\varepsilon\right)\right)=$

$$
\begin{aligned}
& =\sqrt{t} .\left(t \gg \int_{\varepsilon \in I} a(p+\varepsilon)+t \gg \int_{\varepsilon \in I} \delta\left(t^{\circ}+\varepsilon\right)\right)=\sqrt{ } t . t \gg\left(\int_{\varepsilon \in I} a(p+\varepsilon)+\int_{\varepsilon \in I} \delta\left(t^{\circ}+\varepsilon\right)\right)(A T B 3) \\
& =\sqrt{ } t .\left(\int_{\varepsilon \in I} a(p+\varepsilon)+\int_{\varepsilon \in I} \delta\left(t^{\circ}+\varepsilon\right)\right)=\sqrt{ } t .\left(t^{\circ} \gg \int_{\varepsilon \in I} a(p+\varepsilon)+t^{\circ} \gg \int_{\varepsilon \in I} \delta\left(t^{\circ}+\varepsilon\right)\right)
\end{aligned}
$$

We see that in all cases, we obtain

$$
\begin{aligned}
\tilde{a}(p) & =\sqrt{ } t .\left(t^{\circ} \gg \int_{\varepsilon \in I} a(p+\varepsilon)+t^{\circ} \gg \int_{\varepsilon \in I} \delta\left(t^{\circ}+\varepsilon\right)\right)=\sqrt{ } t . t^{\circ} \gg\left(\int_{\varepsilon \in I} a(p+\varepsilon)+\int_{\varepsilon \in I} \delta\left(t^{\circ}+\varepsilon\right)\right)= \\
& =\sqrt{ } t . t^{\circ} \gg \tilde{a}(p) .
\end{aligned}
$$


4.5.6 Proposition: $\tilde{\tilde{a}}+\widetilde{\tilde{\delta}}=\tilde{\tilde{a}}$.

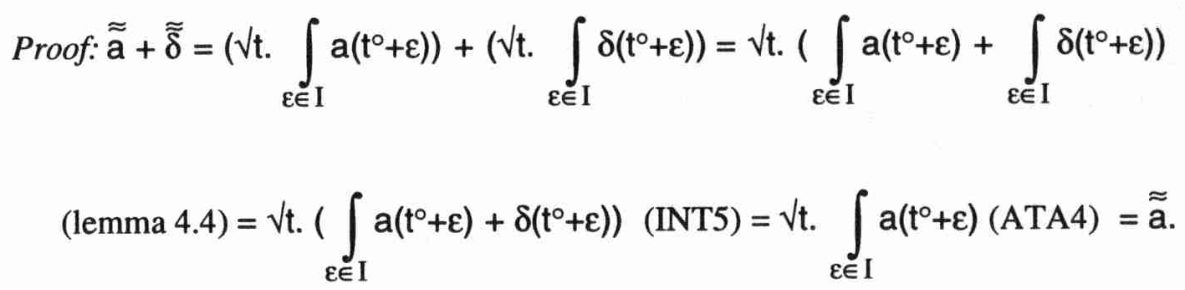

4.5.7 Proposition: $\widetilde{\tilde{a}}=\sqrt{ } t . t^{\circ} \gg \widetilde{\tilde{a}}$.

Proof: $\tilde{a}=\sqrt{t} . \int_{\varepsilon \in I} a\left(t^{\circ}+\varepsilon\right)=\sqrt{ } t . t^{\circ} \gg \int_{\varepsilon \in I} a\left(t^{\circ}+\varepsilon\right)=\sqrt{ } t . t^{\circ} \gg \sqrt{ } u \cdot \int_{\varepsilon \in I} a\left(u^{\circ}+\varepsilon\right)=\sqrt{ } t . t^{\circ} \gg \widetilde{a}$

4.6 Alternative and sequential composition.

We continue with the operators,$+ \cdot$.

4.6.1 Proposition: $(X+\widetilde{\delta})+(Y+\widetilde{\delta})=(X+Y)+\widetilde{\delta}$.

4.6.2 Lemma: $\widetilde{\tilde{\delta}} \cdot \mathrm{X}=\widetilde{\delta}$.

Proof: $\widetilde{\delta} \cdot \mathrm{X}=\left(\sqrt{\mathrm{t}} . \int_{\varepsilon \in \mathrm{I}} \delta\left(\mathrm{t}^{\circ}+\varepsilon\right)\right) \cdot \mathrm{X}=\sqrt{\mathrm{t}} . \int_{\varepsilon \in \mathrm{I}} \delta\left(\mathrm{t}^{\circ}+\varepsilon\right) \cdot \mathrm{X}(\mathrm{IA} 7, \mathrm{INT} 6)=$ $=\sqrt{ }$ t. $\int_{\varepsilon \in \mathrm{I}} \delta\left(\mathrm{t}^{\circ}+\varepsilon\right)(\mathrm{ATA} 2)=\tilde{\delta}$.

4.6.3 Proposition: $(X+\widetilde{\widetilde{\delta}}) \cdot Y=X \cdot Y+\widetilde{\tilde{\delta}}$.

4.6.4 Proposition: $X=\sqrt{ } t . t^{\circ} \gg X \wedge Y=\sqrt{ } t . t^{\circ} \gg Y \Rightarrow(X+Y)=\sqrt{ } t . t^{\circ} \gg(X+Y)$.

4.6.5 Proposition: $\mathrm{X}=\sqrt{\mathrm{t}} \mathrm{t} \mathrm{t}^{\circ} \gg \mathrm{X} \Rightarrow \mathrm{X} \cdot \mathrm{Y}=\sqrt{\mathrm{t}} \mathrm{t} \mathrm{t}^{\circ} \gg \mathrm{X} \cdot \mathrm{Y}$.

4.7 Standard initialisation and left strong choice.

We continue with the operators $\gg_{\mathbf{s}}, \oplus$.

4.7.1 Proposition: $\mathrm{p} \gg_{\mathrm{s}} \mathrm{X}=\mathrm{p} \gg_{\mathrm{s}} \mathrm{X}+\widetilde{\widetilde{\delta}}$.

4.7.2 Proposition: $\mathrm{p} \gg_{\mathrm{s}} \mathrm{X}=\sqrt{\mathrm{t}} \mathrm{t} \mathrm{t}^{\circ} \gg \mathrm{p} \gg_{\mathrm{s}} \mathrm{X}$. 
Proof: $\mathrm{p} \gg_{s} \mathrm{X}=\mathrm{p} \gg \mathrm{X}+\widetilde{\tilde{\delta}}=\mathrm{p} \gg \mathrm{X}+\sqrt{\mathrm{t}}$. $\int_{\varepsilon \in I} \delta\left(\mathrm{t}^{\circ}+\varepsilon\right)=\sqrt{ } \mathrm{t} .\left(\mathrm{t} \gg \mathrm{p} \gg \mathrm{X}+\int_{\varepsilon \in I} \delta\left(\mathrm{t}^{\circ}+\varepsilon\right)\right)$ $(\mathrm{IA} 4)=\sqrt{ } \mathrm{t} .\left(\mathrm{t} \gg \mathrm{t}^{\circ} \gg \mathrm{p} \gg \mathrm{X}+\mathrm{t} \gg \mathrm{t}^{\circ} \gg \int_{\varepsilon \in \mathrm{I}} \delta\left(\mathrm{t}^{\circ}+\varepsilon\right)\right)(\mathrm{SI} 7)=$

$=\sqrt{ } t . t \gg\left(t^{\circ} \gg p \gg X+t^{\circ} \gg \widetilde{\delta}\right)(A T B 3)=\sqrt{ } t .\left(t^{\circ} \gg p \gg X+t^{\circ} \gg \widetilde{\delta}\right)($ lemma 4.4) $=$ $=\sqrt{t} . t^{\circ} \gg(p \gg X+\widetilde{\delta})(A T B 3)=\sqrt{ } t . t^{\circ} \gg\left(p \gg_{s} X\right)$.

4.7.3 Lemma: $\widetilde{\widetilde{\delta}} \oplus \widetilde{\tilde{\delta}}=\widetilde{\delta}$.

Proof: $\widetilde{\delta} \oplus \widetilde{\delta}=\sqrt{ }$ t. $\int_{\varepsilon \in \mathrm{I}} \delta\left(\mathrm{t}^{\circ}+\varepsilon\right) \oplus \sqrt{\mathrm{t}} . \int_{\eta \in \mathrm{I}} \delta\left(\mathrm{t}^{\circ}+\eta\right)=\sqrt{\mathrm{t}} . \int_{\varepsilon \in \mathrm{I}} \delta\left(\mathrm{t}^{\circ}+\varepsilon\right) \oplus \int_{\eta \in \mathrm{I}} \delta\left(\mathrm{t}^{\circ}+\eta\right)$

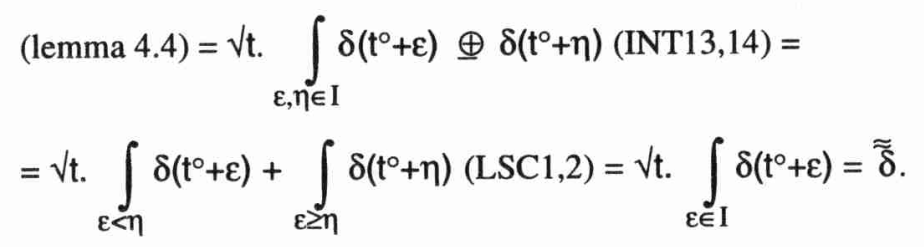

4.7.4 Proposition: $\mathrm{X}=\mathrm{X}+\widetilde{\widetilde{\delta}} \wedge \mathrm{Y}=\mathrm{Y}+\widetilde{\widetilde{\delta}} \Rightarrow \mathrm{X} \oplus \mathrm{Y}=\mathrm{X} \oplus \mathrm{Y}+\widetilde{\widetilde{\delta}}$

4.7.5 Proposition: $X=\sqrt{ }$ t. $\mathrm{t}^{\circ} \gg X \wedge Y=\sqrt{ } t . t^{\circ} \gg Y \Rightarrow X \oplus Y=\sqrt{ } t . t^{\circ} \gg(X \oplus Y)$.

4.8 Parallel composition and encapsulation.

We continue with the operators $\|, \mathbb{L}, \mathrm{I}, \partial_{\mathrm{H}}$.

4.8.1 Lemma: $\mathrm{X}=\mathrm{X}+\widetilde{\delta} \Rightarrow \widetilde{\delta} \amalg \mathrm{X}=\widetilde{\delta}$.

Proof: Assume $X$ satisfies $X=X+\widetilde{\delta}$. We derive

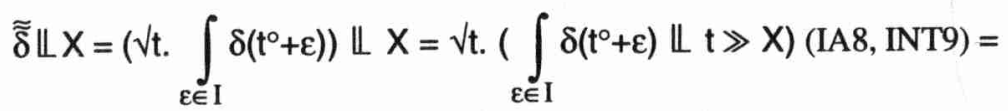
$=\sqrt{t} . \int_{\varepsilon \in \mathrm{I}}\left(\delta\left(t^{\circ}+\varepsilon\right) \oplus \mathrm{t} \gg X\right) \cdot(t \gg X) \quad($ since $t \gg X=0 \gg t \gg X$ by SI 2$)=$ $=\sqrt{ } t . \int_{\varepsilon \in \mathrm{I}}\left(\delta\left(t^{\circ}+\varepsilon\right) \oplus t \gg(X+\widetilde{\delta})\right) \cdot(t \gg X)=$ $=\sqrt{ } t . \int_{\varepsilon \in I}\left(\left(\delta\left(t^{\circ}+\varepsilon\right) \oplus t \gg X\right)+\left(\delta\left(t^{\circ}+\varepsilon\right) \oplus t \gg \tilde{\delta}\right)\right) \cdot(t \gg X)=$ 
$=\sqrt{ } t . \int_{\varepsilon \in I}\left(\left(\delta\left(t^{\circ}+\varepsilon\right) \oplus t \gg X\right)+\delta\left(t^{\circ}+\varepsilon\right)\right) \cdot(t \gg X)=$

$=\sqrt{t} . \int_{\varepsilon \in I} \delta\left(t^{\circ}+\varepsilon\right) \cdot(t \gg X)(\operatorname{SI} 15)=\sqrt{ } t . \int_{\varepsilon \in I} \delta\left(t^{\circ}+\varepsilon\right)(\operatorname{ATA} 2)=\widetilde{\delta}$.

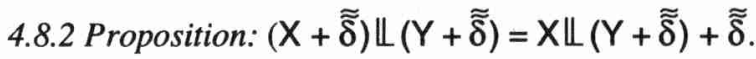

4.8.4 Lemma: $\tilde{\widetilde{\delta}} \mid \widetilde{\tilde{\delta}}=\widetilde{\delta}$.

Proof: $\widetilde{\tilde{\delta}} \mid \widetilde{\delta}=\sqrt{ }$ t. $\int_{\varepsilon \in \mathrm{I}} \delta\left(\mathrm{t}^{\circ}+\varepsilon\right)\left|\sqrt{ } \mathrm{t} . \int_{\eta \in \mathrm{I}} \delta\left(\mathrm{t}^{\circ}+\eta\right)=\sqrt{ } \mathrm{t} . \int_{\varepsilon \in \mathrm{I}} \delta\left(\mathrm{t}^{\circ}+\varepsilon\right)\right| \int_{\eta \in \mathrm{I}} \delta\left(\mathrm{t}^{\circ}+\eta\right)$

(lemma 4.4) $=\sqrt{ }$ t. $\int_{\varepsilon, \eta \in I} \delta\left(t^{\circ}+\varepsilon\right) \mid \delta\left(t^{\circ}+\eta\right)($ INT10, INT11) $=$

$=\sqrt{ } \mathrm{t} . \int_{\varepsilon \in \mathrm{I}} \delta\left(\mathrm{t}^{\circ}+\varepsilon\right)(\mathrm{ATC} 1, \mathrm{ATC} 2)=\widetilde{\delta}$.

4.8.5 Proposition: $X=X+\widetilde{\delta} \wedge Y=Y+\widetilde{\delta} \Rightarrow X|Y=X| Y+\widetilde{\tilde{\delta}}$.

4.8.6 Proposition: $X=X+\widetilde{\delta} \wedge Y=Y+\widetilde{\delta} \Rightarrow X\|Y=X\| Y+\widetilde{\delta}$.

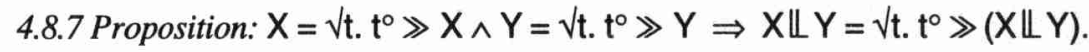

4.8.8 Proposition: $X=\sqrt{ } t . t^{\circ} \gg X \wedge Y=\sqrt{ } t . t^{\circ} \gg Y \Rightarrow X \mid Y=\sqrt{ } t . t^{\circ} \gg(X \mid Y)$.

4.8.9 Proposition: $X=\sqrt{ } t . t^{\circ} \gg X \wedge Y=\sqrt{ } t . t^{\circ} \gg Y \Rightarrow X \| Y=\sqrt{ } t . t^{\circ} \gg(X \| Y)$.

4.8.10 Lemma: $\partial_{\mathrm{H}}(\widetilde{\widetilde{\delta}})=\widetilde{\delta}$.

Proof: $\partial_{H}(\widetilde{\tilde{\delta}})=\partial_{H}\left(\sqrt{ }\right.$ t. $\left.\int_{\varepsilon \in I} \delta\left(t^{\circ}+\varepsilon\right)\right)=\sqrt{ }$ t. $\int_{\varepsilon \in I} \partial_{H}\left(\delta\left(t^{\circ}+\varepsilon\right)\right)($ IA12, INT12) $=$

$=\sqrt{t} . \int_{\varepsilon \in I} \delta\left(t^{\circ}+\varepsilon\right)(D 1)=\tilde{\delta}$.

4.8.11 Proposition: $\partial_{H}(X+\widetilde{\delta})=\partial_{H}(X)+\widetilde{\delta}$.

4.8.12 Proposition: $X=\sqrt{ } t . t^{\circ} \gg X \Rightarrow \partial_{H}(X)=V_{t} . t^{\circ} \gg \partial_{H}(X)$. 
160

4.9 Positive time shift, Shifted initialisation.

Next, the operators $\sigma_{\approx}^{r}, r \gg s t$.

4.9.1 Proposition: $\sigma_{\approx}^{r}(X)=\sigma_{\approx}^{r}(X)+\widetilde{\delta}$.

Proof: Note that since $r \in \mathbb{R}_{>0},(t+r)^{\circ}=t^{\circ}+r>t^{\circ}+\varepsilon$ for all $\varepsilon \in I$. Using this, $\sigma_{\approx}^{r}(X)=\sqrt{ } t .(t+r)^{\circ} \gg X=\sqrt{ } t .\left((t+r)^{\circ} \gg X+\delta\left((t+r)^{\circ}\right)\right)($ SI 5 $)=$

$$
\begin{aligned}
& =\sqrt{t} .\left((t+r)^{\circ} \gg X+\delta\left((t+r)^{\circ}\right)+\int_{\varepsilon \in I} \delta\left(t^{\circ}+\varepsilon\right)\right)(\text { ATA2 })=\sqrt{ } t .\left((t+r)^{\circ} \gg X+\int_{\varepsilon \in I} \delta\left(t^{\circ}+\varepsilon\right)\right) \\
& =\sqrt{t} .\left((t+r)^{\circ} \gg X\right)+\sqrt{ } t . \int_{\varepsilon \in I} \delta\left(t^{\circ}+\varepsilon\right)\left(\text { lemma 4.4) }=\sigma_{\approx}^{r}(X)+\widetilde{\delta} .\right.
\end{aligned}
$$

4.9.2 Proposition: $\sigma_{\approx}^{\mathrm{r}}(\mathrm{X})=\sqrt{ } \mathrm{t} . \mathrm{t}^{\circ} \gg \sigma_{\approx}^{\mathrm{r}}(\mathrm{X})$.

Proof: $\sigma_{\approx}^{r}(\mathrm{X})=\sqrt{\mathrm{t}} \mathrm{t}(\mathrm{t}+\mathrm{r})^{\circ} \gg \mathrm{X}=\sqrt{\mathrm{t}} . \mathrm{t}^{\circ} \gg(\mathrm{t}+\mathrm{r})^{\circ} \gg \mathrm{X}(\mathrm{SI} 2)=\sqrt{\mathrm{t}} \mathrm{t} \mathrm{t}^{\circ} \gg \sqrt{\mathrm{u}} .(\mathrm{u}+\mathrm{r})^{\circ} \gg \mathrm{X}=$ $=\sqrt{t} \cdot t^{\circ} \gg \sigma_{\approx}^{r}(X)$.

4.9.3 Proposition: $r \gg_{s t} X=r \gg_{s t} X+\widetilde{\delta}$.

4.9.4 Proposition: $r \gg_{s t} X=\sqrt{t} . t^{\circ} \gg\left(r \gg_{s t} X\right)$.

Proof: $r \gg_{s t} X=V_{t} .(t+r)^{\circ} \gg t^{\circ} \gg_{s} X=\sqrt{t} . t^{\circ} \gg(t+r)^{\circ} \gg t^{\circ} \gg_{s} X(S I 7)=$ $=\sqrt{t} . t^{\circ} \gg \sqrt{ } u .(u+r)^{\circ} \gg u^{\circ} \gg_{s} X=\sqrt{ } t . t^{\circ} \gg\left(r \gg_{s t} X\right)$.

4.10 Standard initial abstraction.

We consider $\sqrt{S}_{\mathbf{S}}$.

4.10.1 Proposition: $\mathrm{F}=\mathrm{F}+\widetilde{\delta} \Rightarrow V_{\mathrm{s}} \mathrm{p} . \mathrm{F}=\sqrt{\mathrm{S}}_{\mathrm{s}} \mathrm{p} . \mathrm{F}+\widetilde{\delta}$.

Proof: $\sqrt{S}_{s}$ p. $F=V_{s} p .(F+\widetilde{\delta})=\sqrt{ } t .\left(F[t / p]+t^{\circ} \gg \widetilde{\delta}\right)=\sqrt{ } t . F\left[t^{\circ} / p\right]+\sqrt{ } t . t^{\circ} \gg \widetilde{\delta}=$ $=V_{S} p \cdot F+\widetilde{\delta}$.

4.10.2 Proposition: $F=\sqrt{ }$ t. $t^{\circ} \gg F \Rightarrow V_{s} p . F=\sqrt{ } t . t^{\circ} \gg V_{s} p . F$.

Proof: $t \gg V_{s} p . F=t \gg \sqrt{ } u . F\left[u^{\circ} / p\right]=t \gg F[t o / p]=t \gg t^{\circ} \gg F[t / p]=$ $=t \gg\left(\sqrt{ } u \cdot u^{\circ} \gg F\left[u^{\circ} / p\right]\right)=t \gg\left(\sqrt{ } u . u^{\circ} \gg V_{s} p . F\right)$. Now use IA5. 
This finishes the proof that the algebra of standardly initialised processes is closed under all operators of the standard time signature. We proceed to prove additional identities that we will use in the axiomatisations in sections 6 and 7 .

4.11 BPA with urgent actions and absolute time.

First, we prove some identities that allow to derive a standard time variant BPAs $\rho \delta$ of BPAp $\delta$ as defined in 2.2. The familiar equation $X+\widetilde{\delta}=X$ does not hold for all timed processes, as the example $\delta(0)+\widetilde{\delta}=\widetilde{\delta} \neq \delta(0)$ illustrates. This is one reason to limit ourselves to standardly initialised processes. In each case, we will indicate if we need a condition of standardly initialised processes.

4.11.1 Proposition: $\tilde{\delta}(0)=\widetilde{\delta}$.

$$
\begin{aligned}
& \text { Proof: } \tilde{\delta}(0)=\widetilde{\delta}+\int_{\varepsilon \in \mathrm{I}} \delta(\varepsilon)=\left(\sqrt{\mathrm{t}} . \int_{\eta \in \mathrm{I}} \delta\left(\mathrm{t}^{\circ}+\eta\right)\right)+\int_{\varepsilon \in \mathrm{I}} \delta(\varepsilon)= \\
& =\sqrt{ } \mathrm{t} .\left(\int_{\eta \in \mathrm{I}} \delta\left(\mathrm{t}^{\circ}+\eta\right)+\int_{\varepsilon \in \mathrm{I}} \mathrm{t} \gg \delta(\varepsilon)\right) .
\end{aligned}
$$

If $t \gg \delta(\varepsilon)=\delta(t)$, the summand can be dropped by ATA2 since $t<t^{\circ}+\eta$ for some $\eta \in I$; if $\mathrm{t} \gg \delta(\varepsilon)=\delta(\varepsilon)$, the summand can also be dropped by ATA2 since $\varepsilon<\mathrm{t}^{\circ}+2 \varepsilon$. In both cases $\tilde{\delta}(0)=\sqrt{ }$ t. $\left(\int_{\eta \in I} \delta\left(t^{\circ}+\eta\right)+\int_{\varepsilon \in I} t \gg \delta(\varepsilon)\right)=\sqrt{ } t . \int_{\eta \in I} \delta\left(t^{\circ}+\eta\right)=\widetilde{\delta}$.

4.11.2 Proposition: $\tilde{\delta}(p) \cdot X=\tilde{\delta}(p)$.

4.11.3 Proposition: $p<q \Rightarrow \tilde{\delta}(p)+\tilde{\delta}(q)=\tilde{\delta}(q)$.

4.11.4 Proposition: $\tilde{a}(p)+\tilde{\delta}(p)=\tilde{a}(p)$.

4.11.5 Proposition: $X=\sqrt{ } t . t^{\circ} \gg_{s} X \Rightarrow \tilde{a}(p) \cdot X=\tilde{a}(p) \cdot\left(p \gg_{s} X\right)$.

Comment: This is an example of an identity that only holds for standardly initialised processes.

$$
\begin{aligned}
& \text { Proof: } \tilde{a}(p) \cdot X=\left(\widetilde{\delta}+\int_{\varepsilon \in I} a(p+\varepsilon)\right) \cdot X=\tilde{\delta} \cdot X+\int_{\varepsilon \in I} a(p+\varepsilon) \cdot X(I N T 6)= \\
& =\widetilde{\delta}+\int_{\varepsilon \in I} a(p+\varepsilon) \cdot(p+\varepsilon \gg X)(\text { ATA5 })=\tilde{\delta .}\left(p \gg_{s} X\right)+\int_{\varepsilon \in I} a(p+\varepsilon) \cdot\left(p+\varepsilon \gg p \gg_{s} X\right)
\end{aligned}
$$




$$
\text { (assumption) }=\left(\widetilde{\delta}+\int_{\varepsilon \in I} a(p+\varepsilon)\right) \cdot\left(p \gg_{s} X\right)(\text { ATA5, INT6 })=\tilde{a}(p) \cdot\left(p \gg_{s} X\right) .
$$

4.11.6 Proposition: $\mathrm{p} \leq \mathrm{q} \Rightarrow \mathrm{p} \gg_{\mathrm{s}} \tilde{\mathrm{a}}(\mathrm{q})=\tilde{\mathrm{a}}(\mathrm{q})$.

Proof: Suppose $p \leq q$, so $p<q+\varepsilon$ for each $\varepsilon \in I$. Then:

$$
\begin{aligned}
& p \gg_{s} \tilde{a}(q)=\widetilde{\delta}+p \gg\left(\widetilde{\delta}+\int_{\varepsilon \in I} a(q+\varepsilon)\right)=\widetilde{\delta}+p \gg \widetilde{\delta}+\int_{\varepsilon \in I} p \gg a(q+\varepsilon)=\widetilde{\delta}+ \\
& \left.+\int_{\varepsilon \in I} \delta(p+\varepsilon)+\int_{\varepsilon \in I} a(q+\varepsilon)=\widetilde{\delta}+\int_{\varepsilon \in I}(\delta(p+\varepsilon)+a(q+\varepsilon))=\widetilde{\delta}+\int_{\varepsilon \in I} a(q+\varepsilon)\right)=\tilde{a}(q) .
\end{aligned}
$$

4.11.7 Proposition: $p>q \Rightarrow p \gg_{s} \tilde{a}(q)=\tilde{\delta}(p)$.

Proof: Suppose $p>q$, so $p>q+\varepsilon$ for each $\varepsilon \in I$ as $p, q \in \mathbb{R}$. Then:

$$
\begin{aligned}
p \gg_{s} \tilde{a}(q)=\widetilde{\delta}+p \gg\left(\widetilde{\delta}+\int_{\varepsilon \in I} a(q+\varepsilon)\right)=\widetilde{\delta}+p \gg \widetilde{\delta}+\int_{\varepsilon \in I} p \gg a(q+\varepsilon)= \\
\left.=\widetilde{\delta}+\int_{\varepsilon \in I} \delta(p+\varepsilon)+\int_{\varepsilon \in I} \delta(p)=\widetilde{\delta}+\int_{\varepsilon \in I} \delta(p+\varepsilon)+\delta(p)=\widetilde{\delta}+\int_{\varepsilon \in I} \delta(p+\varepsilon)\right)=\tilde{\delta}(p) .
\end{aligned}
$$

4.11.8 Proposition: $\mathrm{p} \gg_{\mathrm{S}}(\mathrm{X}+\mathrm{Y})=\left(\mathrm{p} \gg_{\mathrm{S}} \mathrm{X}\right)+\left(\mathrm{p} \gg_{\mathrm{S}} \mathrm{Y}\right)$.

4.11.9 Proposition: $\mathrm{p} \gg_{\mathrm{s}}(\mathrm{X} \cdot \mathrm{Y})=\left(\mathrm{p} \gg_{\mathrm{s}} \mathrm{X}\right) \cdot \mathrm{Y}$.

4.12 PA with urgent actions and absolute time.

We continue with identities that we will use to axiomatise PAsp $\delta$, the standard time variant of process algebra with free merge (merge without communication).

4.12.1 Proposition: $\mathrm{p} \leq \mathrm{q} \Rightarrow \tilde{\mathrm{a}}(\mathrm{p}) \oplus \tilde{\mathrm{b}}(\mathrm{q})=\tilde{\mathrm{a}}(\mathrm{p})$.

Proof: Straightforward, use INT13, INT14, lemma 4.6.3 and the fact that for each $\varepsilon \in$ I, there is a $\eta \in I$ such that $p+\varepsilon<q+\eta$.

4.12.2 Proposition: $p>q \Rightarrow \tilde{a}(p) \oplus \tilde{b}(q)=\tilde{\delta}(q)$.

Proof: In this case, use that for each $\varepsilon \in \mathrm{I} p+\varepsilon<q$.

4.12.3 Proposition: $X=0 \gg_{s} Z \Rightarrow \tilde{a}(p) \mathbb{X}=(\tilde{a}(p) \oplus X) \cdot X$.

Proof: We consider 3 cases:

Case 1: Let $\varepsilon \in I, r \geq p+\varepsilon$. Then 


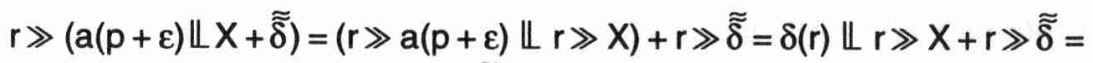
$=(\delta(r) \oplus r \gg X) \cdot(r \gg X)+r \gg \tilde{\delta}($ since $r \gg X=0 \gg r \gg X$ by SI2) $=$ $=\delta(r) \cdot(r \gg X)+r \gg \widetilde{\delta}(S I 16)=\delta(r)+r \gg \widetilde{\delta}=\delta(r) \cdot X+r \gg \widetilde{\delta}=$ $=(r \gg a(p+\varepsilon)) \cdot X+r \gg \widetilde{\delta}=r \gg(a(p+\varepsilon)+a(p+\varepsilon) \oplus X) \cdot X+r \gg \widetilde{\delta}(S I 15)=$ $=r \gg a(p+\varepsilon) \cdot X+r \gg(a(p+\varepsilon) \oplus X) \cdot X+r \gg \widetilde{\delta}=$ $=\delta(r)+r \gg(a(p+\varepsilon) \oplus X) \cdot X+r \gg \widetilde{\delta}=r \gg(a(p+\varepsilon) \oplus X) \cdot X+r \gg \widetilde{\delta}=$ $=r \gg(a(p+\varepsilon) \oplus X) \cdot X+\widetilde{\delta})$.

Case 2: Let $\varepsilon \in I, p \leq r<p+\varepsilon$. Then

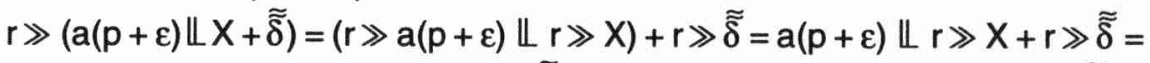
$=(a(p+\varepsilon) \oplus r \gg X) \cdot(r \gg X)+r \gg \widetilde{\delta}$ (as in case 1$)=a(p+\varepsilon) \cdot(r \gg X)+r \gg \widetilde{\delta}$ (since $r \gg X=r \gg(X+\widetilde{\delta})=r \gg X+r \gg \widetilde{\delta}=r \gg X+r \gg \widetilde{\delta}+\delta(p+2 \varepsilon)=$ $=r \gg X+\delta(p+2 \varepsilon))=a(p+\varepsilon) \cdot((p+\varepsilon) \gg r \gg X)+r \gg \widetilde{\delta}=$ $=a(p+\varepsilon) \cdot((p+\varepsilon) \gg X)+r \gg \widetilde{\delta}\left(\right.$ by SI7, since $\left.X=0 \gg_{s} Z\right)=a(p+\varepsilon) \cdot X+r \gg \widetilde{\delta}=$ $=(a(p+\varepsilon) \oplus r \gg X) \cdot X+r \gg \widetilde{\delta}=r \gg(a(p+\varepsilon) \oplus X) \cdot X+r \gg \widetilde{\delta}($ SII $)=$ $=r \gg(a(p+\varepsilon) \oplus X) \cdot X+\widetilde{\widetilde{\delta}})$.

Case 3: Let $\varepsilon \in I, r<p$. Then

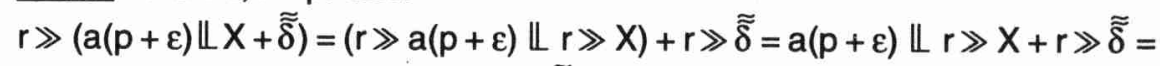
$=(a(p+\varepsilon) \oplus r \gg X) \cdot(r \gg X)+r \gg \widetilde{\delta}$. Now if $a(p+\varepsilon) \oplus r \gg X=a(p+\varepsilon)$, then we proceed as in case 2 . Otherwise, by SI15, $a(p+\varepsilon) \oplus r \gg X$ must be an alternative composition of $\delta$ 's with timestamps less than $p+\varepsilon$. In this case, we proceed as in case 1. We see that we have for all $\varepsilon \in \mathrm{I}, \mathrm{r} \in \mathcal{T}$

$$
r \gg(a(p+\varepsilon) \Perp X+\widetilde{\delta})=r \gg(a(p+\varepsilon) \oplus X) \cdot X+\widetilde{\tilde{\delta}}) .
$$

Now let $r \in \mathcal{T}$. Then:

$\left.r \gg(\tilde{a}(p) \amalg X)=r \gg\left(\widetilde{\delta}+\int_{\varepsilon \in I} a(p+\varepsilon)\right) \Perp X\right)=\int_{\varepsilon \in I} r \gg(\widetilde{\delta} \Perp X+a(p+\varepsilon) \amalg X)$

$$
\begin{aligned}
& \text { (INT2, INT9, IA 16) }=\int_{\varepsilon \in I} r \gg(\tilde{\delta}+a(p+\varepsilon) \amalg X)(\text { lemma 4.8.1) }= \\
& \left.\left.=\int_{\varepsilon \in I} r \gg(a(p+\varepsilon) \oplus X) \cdot X+\widetilde{\delta}\right)=r \gg \int_{\varepsilon \in I}(a(p+\varepsilon) \oplus X) \cdot X+\widetilde{\tilde{\delta}} \cdot X\right) \\
& \left(\text { lemma 4.6.2) }=r \gg \int_{\varepsilon \in I}(a(p+\varepsilon) \oplus X) \cdot X+(\tilde{\tilde{\delta}} \oplus X) \cdot X\right)
\end{aligned}
$$

(use lemma 4.7.3 and SI15) $=r \gg\left(\left(\int_{\varepsilon \in I} a(p+\varepsilon)+\widetilde{\delta}\right) \oplus X\right) \cdot X=$

$=r \gg(\tilde{a}(p) \oplus X) \cdot X$. The proposition now follows by extensionality (IA5).

4.12.4 Proposition: $Y=0 \gg_{s} Z \Rightarrow \tilde{a}(p) \cdot X \| Y=(\tilde{a}(p) \oplus Y) \cdot(X \| Y)$. 


\subsection{Communication and encapsulation.}

Next, we add axioms for the communication merge and encapsulation operator, in order to axiomatise ACPs $\rho$, the standard time variant of ACP $\rho$.

4.13.1 Proposition: $\mathrm{p} \neq \mathrm{q} \Rightarrow \tilde{a}(p) \mid \tilde{b}(q)=\tilde{\delta}(\min (p, q))$.

Proof: Assume without loss of generality $p<q$. Note that since $p, q \in \mathbb{R}$, for all $\varepsilon, \eta \in$ I we have $p+\varepsilon<q+\eta$. Then

$$
\begin{gathered}
\tilde{a}(p)\left|\tilde{b}(q)=\left(\widetilde{\delta}+\int_{\varepsilon \in I} a(p+\varepsilon)\right)\right|\left(\widetilde{\delta}+\int_{\eta \in I}^{b} b(q+\eta)\right)=\widetilde{\delta}+\int_{\varepsilon \in I} \int_{\eta \in I} a(p+\varepsilon) \mid b(q+\eta) \\
\left(\text { INT10, 11) }=\widetilde{\delta}+\int_{\varepsilon \in I} \int_{\eta \in I} \delta(p+\varepsilon)=\widetilde{\delta}+\int_{\varepsilon \in I} \delta(p+\varepsilon)\right)=\tilde{\delta}(p) .
\end{gathered}
$$

4.13.2 Proposition: $\tilde{a}(p) \mid \tilde{b}(p)=(a T b)(p)$.

Proof: $\tilde{a}(p)\left|\tilde{b}(p)=\left(\widetilde{\delta}+\int_{\varepsilon \in I} a(p+\varepsilon)\right)\right|\left(\widetilde{\delta}+\int_{\eta \in I} b(p+\eta)\right)=\widetilde{\delta}+\int_{\varepsilon \in I} \int_{\eta \in I} a(p+\varepsilon) \mid b(p+\eta)$

$$
\left.=\widetilde{\delta}+\int_{\varepsilon \in I} \int_{\eta \in I} a\left|b(p+\varepsilon)=\widetilde{\delta}+\int_{\varepsilon \in I} a\right| b(p+\varepsilon)\right)=(a T b)(p) .
$$

4.13.3 Proposition: $\tilde{a}(p) \cdot X \mid \tilde{b}(q)=(\tilde{a}(p) \mid \tilde{b}(q)) \cdot X$.

4.13.4 Proposition: $\tilde{a}(p) \mid \tilde{b}(q) \cdot X=(\tilde{a}(p) \mid \tilde{b}(q)) \cdot X$.

4.13.5 Proposition: $\tilde{a}(p) \cdot X \mid \tilde{b}(q) \cdot Y=(\tilde{a}(p) \mid \tilde{b}(q)) \cdot(X \| Y)$.

4.13.6 Proposition: $\partial_{H}(\tilde{a}(p))=\left(\partial_{H}(a)\right)(p)$.

\subsection{Standard initial abstraction.}

We present identities used to axiomatise $\mathrm{ACP}_{s \rho} \sqrt{ }$, the standard time variant of $\mathrm{ACP} \rho \sqrt{\text {. }}$

4.14.1 Proposition: $\tilde{a}[p]=\sqrt{ }_{s} q . \tilde{a}(p+q)$. 
Proof: $\tilde{a}[p]=\sqrt{ } t . \int_{\varepsilon \in I} a\left(p+t^{\circ}+\varepsilon\right)=V_{s} q . \quad \int_{\varepsilon \in I} a(p+q+\varepsilon)=V_{s} q . \int_{\varepsilon \in I} a(p+q+\varepsilon)+\delta(q+\varepsilon)$ $=$

$=V_{S} q . \int_{\varepsilon \in I} a(p+q+\varepsilon)+\int_{\varepsilon \in I} \delta(q+\varepsilon)=V_{S} q . \int_{\varepsilon \in I} a(p+q+\varepsilon)+\widetilde{\delta}=V_{S} q \cdot \tilde{a}(p+q)$.

4.14.2 Proposition: $\sqrt{ }_{s} p . F=\sqrt{ }_{s} q . F[q / p]$ if $q$ is not free in $F$.

Proof: Suppose $q$ is not free in $F$. Then

$V_{s} p \cdot F=\sqrt{ } t . F\left[t^{\circ} / p\right]=V_{S} q . F\left[t^{\circ} / p\right]\left[q / t^{\circ}\right]=V_{S} q \cdot F[q / p]$.

4.14.3 Proposition: $p \gg_{S} \sqrt{S}_{S} q \cdot F=p \gg_{S} F[p / q]$.

Proof: $p \gg_{s} V_{s} q . F=p \gg \sqrt{ } t . F[t / q]+\widetilde{\delta}=p \gg F[t \% q][p / t]+\widetilde{\tilde{\delta}}=p \gg F[p / q]+\widetilde{\delta}=$ $=p \gg_{S} F[p / q]$.

4.14.4 Proposition: $\sqrt{ }_{\mathbf{S}} \mathrm{q} . V_{\mathrm{S}} \mathrm{p} . \mathrm{F}=\sqrt{ }_{\mathrm{S}} \mathrm{q} . \mathrm{F}[\mathrm{q} / \mathrm{p}]$.

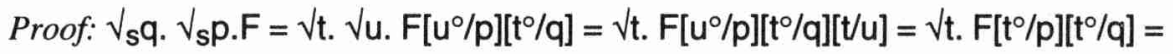
$=\sqrt{t} . F[q / p][t \% q]=V_{s} q \cdot F[q / p]$.

4.14.5 Proposition: $X=\sqrt{s}_{\mathrm{s}} \mathrm{p}$. $\mathrm{X}$.

Proof: $\mathrm{X}=\sqrt{\mathrm{t}} \mathrm{X} \mathrm{X}=\sqrt{\mathrm{s}}_{\mathrm{s}} \mathrm{p} . \mathrm{X}$.

4.14.6 Proposition: $X=\sqrt{t} t t^{\circ} \gg_{S} X \wedge Y=\sqrt{ } t . t^{\circ} \gg_{S} Y \wedge \forall p \in \mathbb{R}_{\geq 0} p \gg_{s} X=p \gg_{S} Y$ $\Rightarrow \mathrm{X}=\mathrm{Y}$.

Proof: Suppose the conditions hold. Let $t \in \mathbb{R}_{\geq 0}+I$. Then

$t \gg X=t \gg\left(\sqrt{ } u \cdot u^{\circ} \gg_{s} X\right)=t \gg\left(t^{\circ} \gg_{s} X\right)=t \gg\left(t^{\circ} \gg_{s} Y\right)=t \gg\left(\sqrt{ } u . u^{\circ} \gg_{s} Y\right)=t \gg Y$.

Now apply extensionality, IA5.

4.14.7 Proposition: $X=\sqrt{t}$. $t^{\circ} \gg_{s} X \Rightarrow\left(V_{s} p \cdot F\right)+X=V_{s} p \cdot\left(F+p \gg_{s} X\right)$.

Proof: $\left(\sqrt{s}_{s}\right.$. F $)+X=\sqrt{ } t . F\left[t^{\circ} / p\right]+\sqrt{t}^{\circ} t^{\circ} \gg_{s} X=\sqrt{ } t .\left(F\left[t^{\circ} / p\right]+t^{\circ} \gg_{s} X\right)=$ $=V_{S} p \cdot\left(F+p \gg_{S} X\right)$.

4.14.8 Proposition: $\left(\bigvee_{s} p . F\right) \cdot X=V_{S} p \cdot(F \cdot X), \partial_{H}\left(V_{S} p \cdot F\right)=V_{s} p . \partial_{H}(F)$

4.14.9 Proposition: $X=\sqrt{t} . t^{\circ} \gg_{s} X \Rightarrow\left(\sqrt{s}_{s} p . F\right) \cup X=\sqrt{s}_{s} p .\left(F \Perp p \gg_{s} X\right)$, $X \mathbb{L}\left(V_{s} p . F\right)=\sqrt{ }_{s} p \cdot\left(p \gg_{s} X \mathbb{L}\right),\left(V_{s} p . F\right) \mid X=V_{s} p \cdot\left(F \mid p \gg_{s} X\right)$, $X \mid\left(V_{s} p . F\right)=V_{s} p .\left(p \gg_{s} X \mid F\right),\left(V_{s} p \cdot F\right) \oplus X=V_{s} p .\left(F \oplus p \gg_{s} X\right)$, $X \oplus\left(V_{s} p . F\right)=V_{s} p \cdot\left(p \gg_{s} X \oplus F\right)$. 
4.15 BPA with urgent actions and relative time.

Now we present identities that will be used in order to directly axiomatize the relative time algebras in section 7 . First, $\mathrm{BPA}_{\text {st }}$.

4.15.1 Proposition: $\sigma \approx_{\approx}^{r} \sigma \underset{\approx}{p}(X)=\sigma_{\approx}^{r+p}(X)$.

Proof: $\sigma_{\approx}^{\mathrm{r}} \circ \sigma \mathrm{\sigma}(\mathrm{X})=\sqrt{\mathrm{t}}$. $\left.(\mathrm{t}+\mathrm{r})^{\circ} \gg\left(\mathrm{Vu} .(\mathrm{u}+\mathrm{p})^{\circ} \gg \mathrm{X}\right)=\sqrt{\mathrm{t}} .(\mathrm{t}+\mathrm{r})^{\circ} \gg\left((\mathrm{t}+\mathrm{r})^{\circ}+\mathrm{p}\right)^{\circ} \gg \mathrm{X}\right)=$ $=\sqrt{ } t .(t+r)^{\circ} \gg(t+r+p)^{\circ} \gg X=\sqrt{ } t .(t+r+p)^{\circ} \gg X(S I 2)=\sigma_{\approx}^{r+p}(X)$.

4.15.2 Proposition: $\sigma_{\approx}^{r}(X)+\sigma_{\approx}^{r}(Y)=\sigma_{\approx}^{r}(X+Y), \sigma_{\approx}^{r}(X) \cdot Y=\sigma_{\approx}^{r}(X \cdot Y)$.

4.16 Left strong choice and shifted initialisation.

4.16.1 Proposition: $\widetilde{\tilde{a}} \oplus \widetilde{\tilde{b}}=\tilde{\tilde{a}}$.

4.16.2 Proposition: $\widetilde{\tilde{a}} \oplus \sigma_{\approx}^{r}(X)=\widetilde{\widetilde{a}}$.

Proof: $\tilde{\tilde{a}} \oplus \sigma_{\approx}^{r}(X)=\sqrt{ } t . \quad \int_{\varepsilon \in I} a\left(t^{\circ}+\varepsilon\right) \oplus \sqrt{t} .(t+r)^{\circ} \gg X=$

$$
\begin{aligned}
& =\sqrt{ } \text { t. } \int_{\varepsilon \in I} a\left(t^{\circ}+\varepsilon\right) \oplus(t+r)^{\circ} \gg X(\text { lemma 4.4) }= \\
& =\sqrt{t} . \int_{\varepsilon \in I} a\left(t^{\circ}+\varepsilon\right) \oplus\left(t^{\circ}+\varepsilon\right) \gg(t+r)^{\circ} \gg X(\text { SI2 })=\sqrt{ } t . \int_{\varepsilon \in I} a\left(t^{\circ}+\varepsilon\right)(\text { SI16 })=\tilde{a} .
\end{aligned}
$$

4.16.3 Proposition: $\sigma_{\approx}^{r}(X) \oplus \widetilde{\tilde{a}}=\widetilde{\delta}$.

Proof: $\sigma_{\approx}^{r}(X) \oplus \widetilde{\widetilde{a}}=\sqrt{ } t .(t+r)^{\circ} \gg X \oplus \sqrt{ } t . \int_{\varepsilon \in I} a\left(t^{\circ}+\varepsilon\right)=$

$=\sqrt{ } t .(t+r)^{\circ} \gg X \oplus \underset{\varepsilon \in I}{\int_{\varepsilon \in I} a\left(t^{\circ}+\varepsilon\right)(\text { lemma 4.4) }=\sqrt{ } t .} \int_{\varepsilon \in I}(t+r)^{\circ} \gg X \oplus a\left(t^{\circ}+\varepsilon\right)$

$(\operatorname{INT} 14)=\sqrt{t} . \int_{\varepsilon \in I}\left(t^{\circ}+\varepsilon\right) \gg(t+r)^{\circ} \gg X \oplus a\left(t^{\circ}+\varepsilon\right)(S I 2)=\sqrt{ } t . \int_{\varepsilon \in I} \delta\left(t^{\circ}+\varepsilon\right)(S I 17)=\tilde{\delta}$

4.16.4 Proposition: $\sigma_{\approx}^{r}(X) \oplus \sigma_{\approx}^{r}(Y)=\sigma_{\approx}^{r}(X \oplus Y)$.

Proof: $\sigma_{\approx}^{r}(X) \oplus \sigma_{\approx}^{r}(Y)=\sqrt{ } t .(t+r)^{\circ} \gg X \oplus \sqrt{ } t .(t+r)^{\circ} \gg Y=$ $=\sqrt{ } \mathrm{t} .(\mathrm{t}+\mathrm{r})^{\circ} \gg(\mathrm{X} \oplus \mathrm{Y})(\mathrm{lemma} 4.4, \mathrm{SI} 14)=\sigma_{\approx}^{\mathrm{r}}(\mathrm{X} \oplus \mathrm{Y})$. 
4.16.5 Proposition: $\mathrm{r} \gg_{\mathrm{st}} \widetilde{\tilde{a}}=\sigma_{\approx}^{r}(\widetilde{\tilde{\delta}})$.

Proof: $r \gg_{s t} \tilde{\tilde{a}}=\sqrt{t} .(t+r)^{\circ} \gg t^{\circ} \gg_{s} \tilde{a}=\sqrt{t} .(t+r)^{\circ} \gg\left(\widetilde{\delta}+\int_{\varepsilon \in I} a\left(t^{\circ}+\varepsilon\right)\right)=$

$$
\begin{aligned}
& =\sqrt{t} .(t+r)^{\circ} \gg \widetilde{\delta}+\int_{\varepsilon \in I}(t+r)^{\circ} \gg a\left(t^{\circ}+\varepsilon\right)= \\
& \left.\left.\left.=\sqrt{t} . \int_{\varepsilon \in I} \delta\left((t+r)^{\circ}+\varepsilon\right)\right)+\delta\left((t+r)^{\circ}\right)=\sqrt{ } t . \int_{\varepsilon \in I} \delta\left((t+r)^{\circ}+\varepsilon\right)\right)=\sqrt{ } t .(t+r)^{\circ} \gg \widetilde{\delta}=\sigma \approx \widetilde{\widetilde{\delta}}\right) .
\end{aligned}
$$

4.16.6 Proposition: $r \gg_{s t}(X+Y)=r \gg_{s t} X+r \gg_{s t} Y$.

4.16.7 Proposition: $\mathrm{r} \gg_{\mathrm{st}}(\mathrm{X} \cdot \mathrm{Y})=\left(\mathrm{r} \gg_{\mathrm{st}} \mathrm{X}\right) \cdot \mathrm{Y}$.

4.16.8 Proposition: $X=X+\widetilde{\delta} \Rightarrow r \gg_{s t} \sigma_{\approx}^{r}(X)=\sigma_{\approx}^{r}(X)$.

Proof: $r \gg_{s t} \sigma_{\approx}^{r}(X)=\sqrt{t} .(t+r)^{\circ} \gg t^{\circ} \gg_{s}(t+r)^{\circ} \gg X=$

$=\sqrt{ } t .(t+r)^{\circ} \gg \widetilde{\delta}+(t+r)^{\circ} \gg t^{\circ} \gg(t+r)^{\circ} \gg X=\sqrt{ } t .(t+r)^{\circ} \gg \widetilde{\delta}+(t+r)^{\circ} \gg X(S I 7, S I 2)=$ $=\sqrt{t} .(t+r)^{\circ} \gg(\tilde{\delta}+X)=\sqrt{ } t .(t+r)^{\circ} \gg X=\sigma_{\approx}^{r}(X)$.

4.16.9 Proposition: $p>r \Rightarrow p \gg_{s t} \sigma_{\approx}^{r}(X)=\sigma_{\approx}^{r}\left((p-r) \gg_{s t} X\right)$.

Proof: $\mathrm{p} \gg_{s t} \sigma_{\approx}^{r}(X)=\sqrt{ } t .(t+p)^{\circ} \gg t^{\circ} \gg_{s}(t+r)^{\circ} \gg X=$

$=\sqrt{ } t .(t+p)^{\circ} \gg \widetilde{\delta}+(t+p)^{\circ} \gg t^{\circ} \gg(t+r)^{\circ} \gg X=\sqrt{ } t .(t+p)^{\circ} \gg \widetilde{\delta}+(t+p)^{\circ} \gg(t+r)^{\circ} \gg X$

$(S I 7)=\sqrt{ } t .(t+p)^{\circ} \gg(t+r)^{\circ} \gg_{s} X=\sqrt{ } t .(t+r)^{\circ} \gg(t+p)^{\circ} \gg(t+r)^{\circ} \gg_{s} X(S I 7)=$ $=\sqrt{t} .(t+r)^{\circ} \gg \sqrt{ } u \cdot(u+p-r)^{\circ} \gg u^{\circ} \gg_{s} X=\sigma_{\approx}^{r}\left((p-r) \gg_{s t} X\right)$.

\subsection{Free merge.}

Next we derive some equations for the left merge in relative standard time, used to axiomatise $\mathrm{PA}_{\text {st. }}$.

4.17.1 Proposition: $X=\sqrt{ } t . t^{\circ} \gg_{s} X \Rightarrow \tilde{a} \amalg X=\tilde{a} \cdot X$.

Proof: Assume (equivalently) that $X$ satisfies $X=X+\widetilde{\delta} \wedge X=\sqrt{ } t . t^{\circ} \gg X$.

Note that $\tilde{a}=\sqrt{ } t . \int_{\varepsilon \in I} a\left(t^{\circ}+\varepsilon\right)=\sqrt{ } t . \int_{\varepsilon \in I} t \gg a\left(t^{\circ}+\varepsilon\right)=\sqrt{ } t . \int_{\varepsilon \in I} a(t+\varepsilon)$. We derive 


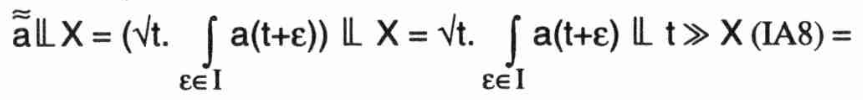
$=\sqrt{t} .\left(\int_{\varepsilon \in I} a(t+\varepsilon) \oplus(t \gg X)\right) \cdot(t \gg X)=\sqrt{ } t .\left(\int_{\varepsilon \in I} a(t+\varepsilon) \oplus(t \gg X+t \gg \widetilde{\delta})\right) \cdot(t \gg X)=$ $=\sqrt{ } t .\left(\int_{\varepsilon \in I} a(t+\varepsilon) \oplus(t \gg X+t \gg \widetilde{\delta}+\delta(t+2 \varepsilon))\right) \cdot(t \gg X)=$ $=\sqrt{ } t \cdot\left(\int_{\varepsilon \in I} a(t+\varepsilon) \oplus(t \gg X)+a(t+\varepsilon) \oplus \delta(t+2 \varepsilon)\right) \cdot(t \gg X)=$ $=\sqrt{ } t .\left(\int_{\varepsilon \in I} a(t+\varepsilon) \oplus(t \gg X)+a(t+\varepsilon)\right) \cdot(t \gg X)=\sqrt{ } t . \int_{\varepsilon \in I} a(t+\varepsilon) \cdot(t \gg X)(S I 15)=$ $=\sqrt{ }$ t. $\int_{\varepsilon \in I} a(t+\varepsilon) \cdot\left(t \gg t^{\circ} \gg X\right)$ (by assumption) $=\sqrt{ } t . \int_{\varepsilon \in I} a(t+\varepsilon) \cdot\left((t+\varepsilon) \gg t \gg t^{\circ} \gg X\right)$ $($ ATA5 $)=\sqrt{ }$ t. $\int_{\varepsilon \in I} a(t+\varepsilon) \cdot\left((t+\varepsilon) \gg t^{\circ} \gg X\right)($ SI7 $)=\sqrt{ } t . \int_{\varepsilon \in I} a(t+\varepsilon) \cdot((t+\varepsilon) \gg X)$ (by assumption, $\left.(t+\varepsilon)^{\circ}=t^{\circ}\right)=\sqrt{ } t . \int_{\varepsilon \in \mathrm{I}} \mathrm{a}(\mathrm{t}+\varepsilon) \cdot \mathrm{X}($ ATA5) $=$ $=\left(\sqrt{ } t . \int_{\varepsilon \in I} a(t+\varepsilon)\right) \cdot X($ INT6, IA7) $=\tilde{\tilde{a}} \cdot X$.

4.17.2 Proposition: $\left.Y=\sqrt{ } t . t^{\circ} \gg_{s} Y \Rightarrow \tilde{\tilde{a}} \cdot \mathrm{X}\right) \amalg \mathrm{L}=\tilde{\tilde{\mathrm{a}}} \cdot(\mathrm{X} \| \mathrm{Y})$.

4.17.3 Proposition: $\sigma_{\approx}^{r}(X) \cup \sigma_{\approx}^{r}(Y)=\sigma_{\approx}^{r}(X \Perp Y)$. Proof: $\sigma_{\approx}^{r}(X) \| \sigma_{\approx}^{r}(Y)=\left(\sqrt{t}\right.$. $\left.(t+r)^{\circ} \gg X\right) \amalg\left(\sqrt{ } t .(t+r)^{\circ} \gg Y\right)=$

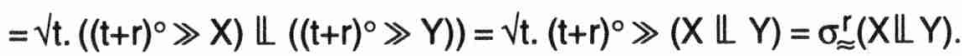

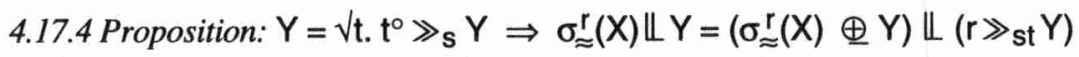

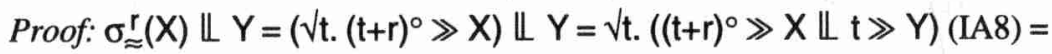
$=\sqrt{t} .\left((t+r)^{\circ} \gg X \oplus t \gg Y\right) \mathbb{L}(t+r)^{\circ} \gg t \gg Y(S I 10)=$ $=\sqrt{ } \mathrm{t} .\left(\mathrm{t} \gg(\mathrm{t}+\mathrm{r})^{\circ} \gg X \oplus \mathrm{X} \mathrm{t} \gg \mathrm{Y}\right) \mathbb{L}(\mathrm{t}+\mathrm{r})^{\circ} \gg t \gg t^{\circ} \gg_{S} \mathrm{Y}(\mathrm{S} \mathrm{S} 2$, assumption $)=$ $=\sqrt{ } t .\left(t \gg \sigma_{\approx}^{r}(X) \oplus t \gg Y\right) \mathbb{L}(t+r)^{\circ} \gg t^{\circ} \gg_{S} Y(S I 7)=$ $=\sqrt{ }$. $t \gg\left(\sigma_{\approx}^{r}(X) \oplus Y\right) \mathbb{t} \gg\left(r \gg_{s t} Y\right)(S I 14)=$ $=\sqrt{t} . t \gg\left(\left(\sigma_{\approx}^{r}(X) \oplus Y\right) \mathbb{L}\left(r \gg_{s t} Y\right)(S I 8)=\right.$ 
$=\left(\sigma_{\approx}^{r}(X) \oplus Y\right) \mathbb{L}\left(r \gg_{\text {st }} Y\right)$ (lemma 4.4, IA4).

\subsection{Merge with communication.}

Now we consider identities concerning parallel composition with communication in relative time, to be used in order to axiomatise $\mathrm{ACP}_{\text {st }}$.

4.18.1 Proposition: $\widetilde{\mathrm{a}} \mid \widetilde{\mathrm{b}}=\mathrm{a} \widetilde{\mathrm{I}} \mathrm{b}$.

$$
\begin{aligned}
& \text { Proof: } \left.\widetilde{\mathrm{a}}\left|\widetilde{\mathrm{b}}=\sqrt{\mathrm{t}} . \int_{\varepsilon \in \mathrm{I}} \mathrm{a}\left(\mathrm{t}^{\circ}+\varepsilon\right)\right| \sqrt{\mathrm{t}} . \int_{\eta \in \mathrm{I}} \mathrm{b}\left(\mathrm{t}^{\circ}+\eta\right)\right)= \\
& =\sqrt{t} . \int_{\varepsilon \in I} a\left(t^{\circ}+\varepsilon\right) \mid \int_{\eta \in I} b\left(t^{\circ}+\eta\right) \quad \text { (lemma 4.7.3) }= \\
& \left.=\sqrt{t} . \int_{\varepsilon \in \mathrm{I}} \int_{\eta \in \mathrm{I}} \mathrm{a}\left(\mathrm{t}^{\circ}+\varepsilon\right)\right) \mid \mathrm{b}\left(\mathrm{t}^{\circ}+\eta\right) \quad \text { (INT10, INT11) }= \\
& =\sqrt{ } \text { t. } \int_{\varepsilon \in \mathrm{I}} \int_{\eta \in \mathrm{I}} \delta\left(\mathrm{t}^{\circ}+\varepsilon\right)+(\mathrm{a} \mid \mathrm{b})\left(\mathrm{t}^{\circ}+\eta\right)+\delta\left(\mathrm{t}^{\circ}+\eta\right) \\
& \text { (ATC1, ATC2, } \\
& \text { (NT2) }= \\
& =\sqrt{ } \mathrm{t} . \int_{\eta \in \mathrm{I}}(\mathrm{a} \mid \mathrm{b})\left(\mathrm{t}^{\circ}+\eta\right) \quad(\text { ATA3, INT2 })=\mathrm{a} \widetilde{\mathrm{I}} \mathrm{b} .
\end{aligned}
$$

4.18.2 Proposition: $(\widetilde{\tilde{a}} \cdot \mathrm{X})|\widetilde{\mathrm{b}}=(\widetilde{\tilde{\mathrm{a}}} \mid \widetilde{\mathrm{b}}) \cdot \mathrm{X}, \widetilde{\tilde{\mathrm{a}}}|(\widetilde{\widetilde{\mathrm{b}}} \cdot \mathrm{X})=(\widetilde{\tilde{\mathrm{a}}} \mid \widetilde{\mathrm{b}}) \cdot \mathrm{X}$, $(\tilde{\tilde{a}} \cdot X) \mid(\widetilde{\mathrm{b}} \cdot Y)=(\tilde{\mathrm{a}} \mid \tilde{\mathrm{b}}) \cdot(X \| Y)$.

4.18.3 Proposition: $\widetilde{\tilde{a}} \mid \sigma \approx r(X)=\widetilde{\delta}$.

Proof: $\tilde{a}\left|\sigma_{\approx}^{r}(X)=\sqrt{ } t . \int_{\varepsilon \in I} a\left(t^{\circ}+\varepsilon\right)\right| \sqrt{ } t .(t+r)^{\circ} \gg X=\sqrt{ } t . \int_{\varepsilon \in I} a\left(t^{\circ}+\varepsilon\right) \mid(t+r)^{\circ} \gg X$

$$
\begin{aligned}
& \text { (lemma 4.4)= لvt. } \int_{\varepsilon \in I} a\left(t^{\circ}+\varepsilon\right) \mid\left(t^{\circ}+\varepsilon\right) \gg(t+r)^{\circ} \gg X(\text { SI2 })=\sqrt{ } t . \int_{\varepsilon \in I} \delta\left(t^{\circ}+\varepsilon\right)(\text { SI12) } \\
& =\widetilde{\delta} .
\end{aligned}
$$

4.18.4 Proposition: $\sigma_{\approx}^{r}(X)|\widetilde{\tilde{a}}=\widetilde{\delta},(\tilde{\tilde{a}} \cdot X)| \sigma_{\approx}^{r}(Y)=\tilde{\delta}, \sigma_{\approx}^{r}(X) \mid(\tilde{a} \cdot Y)=\tilde{\delta}$. 
4.18.5 Proposition: $\sigma_{\approx}^{r}(X) \mid \sigma_{\approx}^{r}(Y)=\sigma_{\approx}^{r}(X \mid Y)$.

Proof: $\sigma_{\approx}^{r}(\mathrm{X})\left|\sigma_{\approx}^{\mathrm{r}}(\mathrm{Y})=\sqrt{\mathrm{t}} \mathrm{t}(\mathrm{t}+\mathrm{r})^{\circ} \gg \mathrm{X}\right| \mathrm{V}_{\mathrm{t}} .(\mathrm{t}+\mathrm{r})^{\circ} \gg \mathrm{Y}=\sqrt{\mathrm{t}} \mathrm{.}(\mathrm{t}+\mathrm{r})^{\circ} \gg \mathrm{X} \mid(\mathrm{t}+\mathrm{r})^{\circ} \gg \mathrm{Y}$

$\left(\right.$ lemma 4.4) $=\sqrt{ }$ t. $(t+r)^{\circ} \gg(X \mid Y)(S I 9)=\sigma_{\approx}^{r}(X \mid Y)$.

\subsection{Encapsulation.}

We continue with equations for the encapsulation operator in relative time, to be used for $\mathrm{ACP}_{\text {st }}$.

4.19.1 Proposition: $\partial_{\mathrm{H}}(\widetilde{\tilde{\mathrm{a}}})=\widetilde{\tilde{\mathrm{a}}}$ if $\mathrm{a} \notin \mathrm{H}$.

Proof: $\partial_{H}(\tilde{\tilde{a}})=\partial_{H}\left(\sqrt{ } t . \int_{\varepsilon \in I} a\left(t^{\circ}+\varepsilon\right)\right)=\sqrt{ } t . \partial_{H}\left(\int_{\varepsilon \in I} a\left(t^{\circ}+\varepsilon\right)\right)(I A 12)=$

$=\sqrt{t} . \int_{\varepsilon \in I} \partial_{H}\left(a\left(t^{\circ}+\varepsilon\right)\right)(I N T 12)=\sqrt{ } t . \int_{\varepsilon \in I} a\left(t^{\circ}+\varepsilon\right)(D 1)=\tilde{a}$.

4.19.2 Proposition: $\partial_{\mathrm{H}}(\widetilde{\tilde{a}})=\widetilde{\tilde{\delta}}$ if $\mathrm{a} \in \mathrm{H}$.

Proof: $\partial_{H}(\widetilde{\tilde{a}})=\partial_{H}\left(\sqrt{ } t . \int_{\varepsilon \in I} a\left(t^{\circ}+\varepsilon\right)\right)=\sqrt{ } t . \partial_{H}\left(\int_{\varepsilon \in I} a\left(t^{\circ}+\varepsilon\right)\right)(I A 12)=$

$=\sqrt{t} . \int_{\varepsilon \in I} \partial H\left(a\left(t^{\circ}+\varepsilon\right)\right)(I N T 12)=\sqrt{ } t . \int_{\varepsilon \in I} \delta\left(t^{\circ}+\varepsilon\right)(D 2)=\widetilde{\delta}$.

4.19.3 Proposition: $\partial_{H}\left(\sigma_{\approx}^{r}(X)\right)=\sigma_{\approx}^{r}\left(\partial_{H}(X)\right)$.

Proof: $\partial_{H}\left(\sigma_{\approx}^{r}(X)\right)=\partial_{H}\left(\sqrt{ } t .(t+r)^{\circ} \gg X\right)=\sqrt{ } t . \partial_{H}\left((t+r)^{\circ} \gg X\right)(I A 12)=$ $=\sqrt{t} .(t+r)^{\circ} \gg \partial_{H}(X)(S I 13)=\sigma_{\approx}^{r}\left(\partial_{H}(X)\right)$.

4.20 Embedding standard relative time into standard absolute time with initial abstraction.

We finish this section with some identities that are used to embed $\mathrm{BPA}_{\delta s t}$ into $B P A_{s \rho} \sqrt{\text {, }}$ $\mathrm{PA}_{s t}$ into $\mathrm{PA}_{\mathrm{s} \rho} \sqrt{ }$ and $\mathrm{ACP}_{\text {st }}$ into $\mathrm{ACP}_{\mathrm{s} \rho} \vee$.

4.20.1 Proposition: $\tilde{\tilde{a}}=V_{s} p \cdot \tilde{a}(p)$.

4.20.2 Proposition: $\sigma_{\approx}^{r}(X)=V_{s} p .(p+r) \gg_{s} X$.

Proof: $\sigma_{\approx}^{r}(X)=\sqrt{ } t .(t+r)^{\circ} \gg X=\sqrt{ } t .\left(\delta\left((t+r)^{\circ}\right)+(t+r)^{\circ} \gg X\right)($ SI5 $)=$ 


$$
\begin{aligned}
& =\sqrt{t} \cdot\left(\int_{\varepsilon \in I} \delta\left(t^{\circ}+\varepsilon\right)+\delta\left((t+r)^{\circ}\right)+(t+r)^{\circ} \gg X\right)=\sqrt{ } t .\left(\int_{\varepsilon \in I} \delta\left(t^{\circ}+\varepsilon\right)+(t+r)^{\circ} \gg X\right)= \\
& =\sqrt{ } t .\left(\widetilde{\delta}+(t+r)^{\circ} \gg X\right)=V_{s} p .(\widetilde{\delta}+(p+r \gg X))=V_{s} p \cdot(p+r) \gg_{s} X .
\end{aligned}
$$

4.20.3 Proposition: $r \gg_{s t} X=V_{s} p .(p+r) \gg_{s} p \gg_{s} X$.

$$
\begin{aligned}
& \text { Proof: } r \gg_{s t} X=\sqrt{ } t .(t+r)^{\circ} \gg^{\circ} \gg_{s} X=\sqrt{t} . \delta\left((t+r)^{\circ}\right)+(t+r)^{\circ} \gg^{\circ} \gg_{s} X(S I 5)= \\
& =\sqrt{ } t . \int_{\varepsilon \in I} \delta\left(t^{\circ}+\varepsilon\right)+\delta\left((t+r)^{\circ}\right)+(t+r)^{\circ} \gg^{\circ} \gg_{s} X=\sqrt{ } t . \tilde{\delta}+(t+r)^{\circ} \gg^{\circ} \gg_{s} X= \\
& =\sqrt{ } t .(t+r)^{\circ} \gg_{s} t^{\circ} \gg_{s} X=V_{s} p .(p+r) \gg_{s} p \gg_{s} X .
\end{aligned}
$$

\section{Standardised processes.}

In section 2, we have sketched a theory ACP $\rho \sqrt{I}$ and a model $M_{A}^{\star}$ that contain both standard real time and nonstandard real time processes. In section 4 , we concentrated on the subalgebra of standardly initialised processes. In this section, we will concentrate on the further subalgebra and the model generated by the standard time processes. For this reason, we introduce the standardisation operator $S$.

\subsection{Definition.}

First, we define standardisation as an operator on $\mathrm{P}^{\star}$. We have the axioms in table 7.

$$
\begin{array}{ll}
S(\mathrm{a}(\mathrm{t}))=\tilde{\mathrm{a}}\left(\mathrm{t}^{\circ}\right) & \\
S(\mathrm{X}+\mathrm{Y})=S(\mathrm{X})+\mathcal{S}(\mathrm{Y}) & S(\mathrm{X} \cdot \mathrm{Y})=S(\mathrm{X}) \cdot S(\mathrm{Y}) \\
S\left(\int_{\mathrm{v} \in \mathrm{V}} \mathrm{F}\right)=\int_{\mathrm{v} \in \mathrm{V}} S(\mathrm{~F}) & S(\sqrt{ } \mathrm{t} . \mathrm{F})=\sqrt{ } \mathrm{t} . S(\mathrm{~F})
\end{array}
$$

TABLE 7. Standardisation.

We give some examples:
1. $S(\delta)=\tilde{\delta}$
2. $S(\tilde{a}[p])=\tilde{a}[p]$
3. $\mathcal{S}(\tilde{a}(p))=\tilde{a}(p)$.

In the model, $S(\mathrm{p})$ is obtained by replacing each transition $\left.\langle s, t\rangle^{a(r)} \overrightarrow{r^{\prime}}, r\right\rangle$ by transitions $\langle s, t\rangle \stackrel{a(v)}{\longrightarrow}\left\langle s^{\prime}, v\right\rangle$ for each $v>t$ of the form $v=r^{\circ}+\varepsilon$ for some $\varepsilon \in I$, and if $\langle s, t\rangle$ is a state without outgoing transitions and $s \neq \sqrt{ }$, then we add transitions $\langle s, t\rangle \rightarrow$ $\langle s, r\rangle$ for each $r>t$ of the form $r=t^{\circ}+\varepsilon$ for some $\varepsilon \in I$ and also transitions $\left\langle s^{\prime}, t^{\prime}\right\rangle \rightarrow$ $\langle s, r\rangle$ (for the same r's) for each transition $\left\langle s^{\prime}, t^{\prime}\right\rangle \rightarrow\langle s, t\rangle$. 


\subsection{Properties.}

1. $S$ does not distribute over $\|, \mathbb{L}, \mathrm{I}$.

2. $S$ is a projection: $S(S(X))=S(X)$.

3. $S\left(\partial_{H}(X)\right)=\partial_{H}(S(X))$

Proof: We only prove 1. Consider $\|$. Let $\varepsilon \in \mathrm{I}$.

$S(a(1+\varepsilon) \| b(1+2 \varepsilon))=S(a(1+\varepsilon) \cdot b(1+2 \varepsilon))=\tilde{a}(1) \cdot \tilde{b}(1)=\tilde{a}(1) \cdot \tilde{b}$, but

$$
\begin{aligned}
& S(a(1+\varepsilon))\|S(b(1+2 \varepsilon))=\tilde{a}(1)\| \tilde{b}(1)=\tilde{a}(1) \cdot \tilde{b}(1)+a \tau b(1)+\tilde{b}(1) \cdot \tilde{a}(1)= \\
& \quad=\tilde{a}(1) \cdot \tilde{b}+a \top b(1)+\tilde{b}(1) \cdot \tilde{a} .
\end{aligned}
$$

\subsection{Definition.}

We say that a process $\mathrm{X}$ is standardised, $\mathrm{X} \in \mathrm{SIS}$, if $\mathcal{S}(\mathrm{X})=\mathrm{X}$. Also, we define this concept directly on the model $M_{A}^{*}$. Let $f \in M_{A}^{*}$. Then $f$ is standardised, $f \in S\left(M_{A}^{*}\right)$, if:

i. for all $t \in \mathbb{R}_{\geq 0}+I$, all actions of $f(t)$ have a timestamp not in $\mathbb{R}_{\geq 0}$.

ii. for all $t \in \mathbb{R}_{\geq 0}+\mathrm{I}$ we have the following: let $\langle p, v\rangle \stackrel{\mathrm{a}(r)}{\longrightarrow}\langle q, r\rangle$ be a transition in $f(t)$. Then:

a. for each $r^{\prime}=r+\varepsilon$ for some $\varepsilon \in I$ there is a $q^{\prime}$ with $\langle p, v\rangle \stackrel{a\left(r^{\prime}\right)}{\longrightarrow}\left\langle q^{\prime}, r^{\prime}\right\rangle$ and $\left\langle q, r^{\prime}\right\rangle \leftrightarrows$ $\left\langle q^{\prime}, r^{\prime}\right\rangle$;

b. for each $r^{\prime}$ with $r=r^{\prime}+\varepsilon$ for some $\varepsilon \in I$ and $r^{\prime}>v$ there is a $q^{\prime}$ with $\langle p, v\rangle \stackrel{a\left(r^{\prime}\right)}{\rightarrow}\left\langle q^{\prime}\right.$, $\left.r^{\prime}\right\rangle$ and $\langle q, r\rangle \leftrightarrow\left\langle q^{\prime}, r\right\rangle$.

iii. similarly for idle transitions: let $\langle p, v\rangle \rightarrow\langle q, r\rangle$ be a transition in $f(t)$. Then:

a. for each $r^{\prime}=r+\varepsilon$ for some $\varepsilon \in I$ there is a $q^{\prime}$ with $\langle p, v\rangle \rightarrow\left\langle q^{\prime}, r^{\prime}\right\rangle$ and $\left\langle q, r^{\prime}\right\rangle \leqq\left\langle q^{\prime}\right.$, r');

b. for each $r^{\prime}$ with $r=r^{\prime}+\varepsilon$ for some $\varepsilon \in I$ and $r^{\prime}>v$ there is a $q^{\prime}$ with $\langle p, v\rangle \rightarrow\left\langle q^{\prime}, r^{\prime}\right\rangle$ and $\langle q, r\rangle \leftrightarrows\left\langle q^{\prime}, r\right\rangle$.

\subsection{Proposition.}

1. $\tilde{a}(p), \tilde{a}[p] \in S I S, a(t) \notin S I S$.

2. $X \in S I S \Rightarrow \partial_{H}(X), \sigma_{\approx}^{r}(X), r \gg_{S t} X \in S I S$.

3. $X, Y \in S I S \Rightarrow X+Y, X \cdot Y, X\|Y, X\| Y, X \mid Y, X \oplus Y \in D I S$.

4. for all $v \in V F \in S I S \Rightarrow \int_{v \in V} F \in$ SIS.

5. $X \in S I S \Rightarrow X \in S I P$

6. $V t . a\left(t^{\circ}+1\right) \in S I P-S I S$.

Proof: We only prove 6. $S\left(\sqrt{ } t . a\left(t^{\circ}+1\right)\right)=\sqrt{ } t . S\left(a\left(t^{\circ}+1\right)=\sqrt{ } t . \tilde{a}\left(t^{\circ}+1\right)=\tilde{a}[1] \neq\right.$ $\neq \sqrt{t}$. $a\left(t^{\circ}+1\right)$. For the positive result, see 4.3 . 
Note: 5 ensures that all equations in section 4 hold for all standardised processes. Also notice that a deadlock possibility may not be preserved by standardisation: the process $\mathrm{a}(1+\varepsilon)+\delta(1+2 \varepsilon)(\varepsilon \in \mathrm{I})$ has a deadlock possibility, but its standardisation does not.

\subsection{A graph model with actions and time separated.}

Consider $S\left(\mathrm{M}_{\mathrm{A}}^{*}\right)$, the collection of processes that satisfy $S(\mathrm{X})=\mathrm{X}$. This collection of processes is also closed under all elements of the standard time signature. This indicates the existence of an interesting subalgebra with domain $S\left(M_{A}^{*}\right)$ of the reduct of $M_{A}^{*}$ to the standard time signature.

We obtain a subalgebra (of a reduct of) $M_{A}^{*}$ with domain $S\left(M_{A}^{*}\right)$ and signature $\Sigma(\mathrm{ST})$. In the following section 6 , we will discuss a direct axiomatisation of this algebra. In section 7, we will present a direct axiomatisation of the elements of this algebra that have only relative time notation (a further restriction to the signature $\left.\Sigma\left(\mathrm{ACP}_{\text {st }}\right)\right)$. Here, we present a simplified presentation of the subalgebra using transition systems with actions and time separated. This is the model $G_{A}^{*}$ that we will use in sections 6 and 7 .

We define a set of process graphs as in [BAW90] with labels from $A \cup \mathbb{R}_{>0}$ satisfying two extra conditions:

i. every node has at most one outgoing s-labeled edge $\left(s \in \mathbb{R}_{>0}\right)$;

ii. an s-labeled edge $\left(s \in \mathbb{R}_{>0}\right)$ may not lead to a termination node.

Let $G_{A}^{*}$ be the set of such process graphs with cardinality $\leq 2{ }^{N_{0}}$. To state this precisely, an element of $G_{A}^{*}$ is a quadruple $\langle\mathrm{N}, \mathrm{E}, \mathrm{r}, \mathrm{T}\rangle$ where $\mathrm{N}$ is the set of nodes, $\mathrm{E} \subseteq \mathrm{N} \times$ $A \cup \mathbb{R}_{>0} \times \mathrm{N}$ is the set of edges, $\mathrm{r} \in \mathrm{N}$ is the root node, and $\mathrm{T} \subseteq \mathrm{N}$ is the set of termination nodes. We will always have that a termination node has no outgoing edges. A node without outgoing edges that is not a termination node is called a deadlock node.

We define a mapping from $S\left(\mathrm{M}_{\mathrm{A}}^{*}\right)$ to $G_{\mathrm{A}}^{*}$ by defining a mapping $\phi$ from real time transition systems to process graphs. Let $R$ be a real time transition system. For simplicity, we assume that $R$ is actually a tree, so each node has at most one incoming transition. The set of states of $\phi(R)$ consists of those states of $R$ with time coordinate in $\mathbb{R}_{\geq 0}$. The root of $\phi(R)$ is the root of $R$. Now we consider the transitions. If $\langle s, t\rangle \rightarrow$ $\left\langle s^{\prime}, t^{\prime}\right\rangle$ is a transition in $R$ and $t^{\circ}-t^{\circ}=p>0$, then we have a transition $\left\langle s, t^{\circ}\right\rangle \stackrel{p}{\rightarrow}\left\langle s^{\prime}\right.$, $\left.t^{\prime}\right\rangle$ in $\phi(R)$. If $\left\langle s, t^{\circ}\right\rangle^{a(t)} \rightarrow\left\langle s^{\prime}, t\right\rangle$ is a transition in $R$ (note that for standardised processes always $t^{\circ}\left\langle t\right.$ for any $a(t)$-transition), then we have a transition $\left\langle s, t^{\circ}\right\rangle \stackrel{a}{\rightarrow}\left\langle s^{\prime}\right.$, $\left.t^{\circ}\right\rangle$ in $\phi(R)$.

This describes $\phi$. We then obtain a mapping from $S\left(M_{A}^{*}\right)$, to $G_{A}^{*}$ by mapping a representative of $f \in \mathcal{S}\left(M_{A}^{*}\right)$ to $R(f(0)) / \leftrightarrow$. 
The inverse mapping can be defined along the same lines. We leave the verification that this indeed defines an isomorphism to the reader.

\subsection{Note.}

Thus, we have obtained a series of algebras $M_{A}^{*}$, si $_{-} M_{A}^{*}, S\left(M_{A}^{*}\right), G_{A}^{*}$. We can also consider the subalgebras obtained if we look at absolute time notation processes only, i.e. processes $X$ satisfying $X=0 \gg Z$ for some $Z$. This restriction gives us algebras $M_{A}$, si- $M_{A}, S\left(M_{A}\right), G_{A}$. On the other hand, we can look at processes involving relative time notation only. We do this as in [2].

\subsection{Definition.}

We have the positive time shift operator $\sigma_{+}^{r}$ introduced in [8] with the equations of [2], e.g. $\sigma_{+}^{r}(a(t))=a(t+r)$. On $M_{A}^{*}$, this operator is obtained by incrementing each time stamp of a state or an action in a transition system by $r$.

\subsection{Definition.}

A process $X$ is translatable if for all $r \geq 0$ we have $r \gg X=\sigma_{+}^{r}(0 \gg X)$. A process $X$ is standardly translatable if for all $r \in \mathbb{R}_{>0}$ we have $r \gg_{s} X=\sigma_{+}^{r}\left(0 \gg_{s} X\right)$.

\subsection{Remarks.}

1. Let $X$ be translatable and let $t=r+s$. Then $t \gg X=\sigma_{+}^{r}\left(\sigma_{+}^{s}(X)\right)=\sigma_{+}^{r}(s \gg X)$.

2. $\tilde{a}(p)$ is not standardly translatable, since $2 \gg_{s} \tilde{a}(5)=\tilde{a}(5)$ but $\sigma_{+}^{2}\left(0 \gg_{s} \tilde{a}(5)\right)=$ $=\sigma_{+}^{2}(\tilde{a}(5))=\tilde{a}(7)$.

3. $\tilde{a}[p]$ is standardly translatable, since $r \gg_{s} \tilde{a}[p]=\tilde{a}[p+r]=\sigma_{+}^{r}(\tilde{a}(p))=\sigma_{+}^{r}\left(0 \gg_{s} \tilde{a}[p]\right)$.

4. $X \in$ si- $M_{A}^{\star}$, then $X$ is translatable iff $X$ is standardly translatable.

\subsection{Algebras.}

We obtain four more algebras. $M_{A}^{r}$ is the subalgebra of $M_{A}^{*}$ consisting of all translatable processes, si- $M_{A}^{r}$ is the subalgebra of si- $M_{A}^{*}$ consisting of all standardly translatable processes, $S\left(M_{A}^{r}\right)$ is the subalgebra of $S\left(M_{A}^{*}\right)$ consisting of all its standardly translatable processes, $G_{A}^{r}$ is the subalgebra of $G_{A}^{*}$ consisting of all its standardly translatable processes. $G_{A}^{r}$ is a model for $\mathrm{ACP}_{\text {st }}$, the theory of relative time standard time process algebra (to be defined in 7.13), while all laws used to axiomatise $\mathrm{ACP}_{\text {st }}$ are valid in si$M_{A}^{r}$. We give an overview of all algebras introduced in fig. 2. Again, rectangular boxes denote absolute time, boxes with rounded corners denote relative time. 


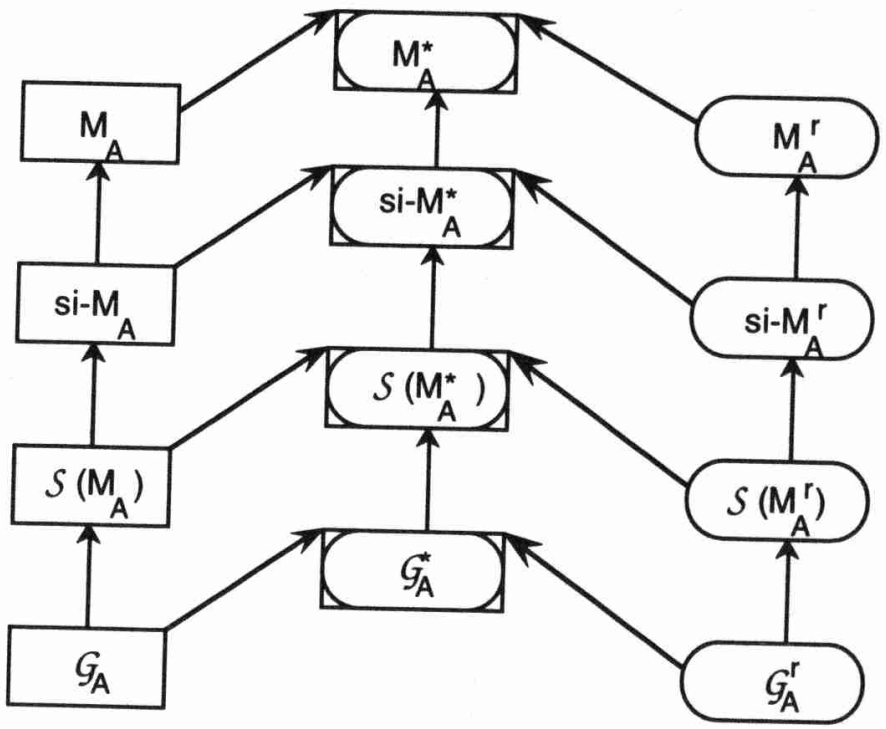

FIGURE 2.

\section{Standard real time process algebra: absolute time.}

\subsection{Basic process algebra.}

The process $\tilde{a}(p)$ can let time progress up to and including $p$, will then execute action $a$ at time $p$, and then terminate successfully. The process $\tilde{\delta}(p)$ can also let time progress up to and including $p$, but then nothing more is possible (in particular, time cannot progress anymore).

Standard Real Time Basic Process Algebra with Deadlock (BPAs $\rho \delta$ ) is the variant

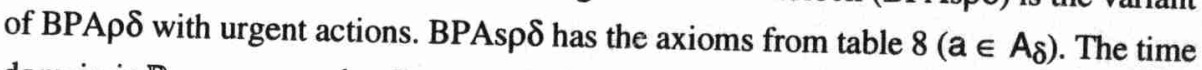
domain is $\mathbb{R}_{\geq 0}$, so we only allow standard real numbers.

- constants $\widetilde{\tilde{\delta}}$

$$
\tilde{a}(p)
$$

- functions $+: P \times P \rightarrow P$

$\therefore \mathrm{P} \times \mathrm{P} \rightarrow \mathrm{P}$

$\gg_{\mathrm{S}}: \mathbb{R}_{\geq_{0}} \times \mathrm{P} \rightarrow \mathrm{P}$ inaction action $(a \in A \cup\{\delta\})$ alternative composition sequential composition standard initialisation 


$$
\begin{array}{ll}
X+Y=Y+X & X=X+\tilde{\delta} \\
(X+Y)+Z=X+(Y+Z) & \tilde{\delta} \cdot X=\tilde{\delta} \\
X+X=X & \\
(X+Y) \cdot Z=X \cdot Z+Y \cdot Z & \\
(X \cdot Y) \cdot Z=X \cdot(Y \cdot Z) & \\
\tilde{\delta}(0)=\tilde{\delta} & p \leq q \Rightarrow p \gg_{s} \tilde{a}(q)=\tilde{a}(q) \\
\tilde{\delta}(p) \cdot X=\tilde{\delta}(p) & p>q \Rightarrow p \gg_{s} \tilde{a}(q)=\tilde{\delta}(p) \\
p<q \Rightarrow \tilde{\delta}(p)+\tilde{\delta}(q)=\tilde{\delta}(q) & p \gg_{s}(X+Y)=\left(p \gg_{s} X\right)+\left(p \gg_{s} Y\right) \\
\tilde{a}(p)+\tilde{\delta}(p)=\tilde{a}(p) & p \gg_{s}(X \cdot Y)=\left(p \gg_{s} X\right) \cdot Y \\
\tilde{a}(p) \cdot X=\tilde{a}(p) \cdot\left(p \gg_{s} X\right) &
\end{array}
$$

TABLE 8. BPAs $\rho \delta$.

\subsection{Operational semantics.}

The operational semantics is defined on the domain of process graphs defined in 5.5 . We have action rules with two types of transitions:

idle $\subseteq P \times \mathbb{R}_{\geq 0} \times \mathbb{R}_{>0} \times P \times \mathbb{R}_{\geq 0}$, notation $\langle x, p\rangle \stackrel{r}{\rightarrow}\left\langle x^{\prime}, p^{\prime}\right\rangle$ step $\subseteq P \times \mathbb{R}_{\geq 0} \times A \times P \times \mathbb{R}_{\geq 0}$, notation $\langle x, p\rangle \stackrel{a}{\rightarrow}\left\langle x^{\prime}, p^{\prime}\right\rangle$.

If $\langle x, p\rangle \stackrel{r}{\rightarrow}\left\langle x^{\prime}, p^{\prime}\right\rangle$, then $r>0, x=x^{\prime}$ and $p^{\prime}=p+r$; if $\langle x, p\rangle \stackrel{a}{\rightarrow}\left\langle x^{\prime}, p^{\prime}\right\rangle$, then $a \in A$ and $p^{\prime}=p$.

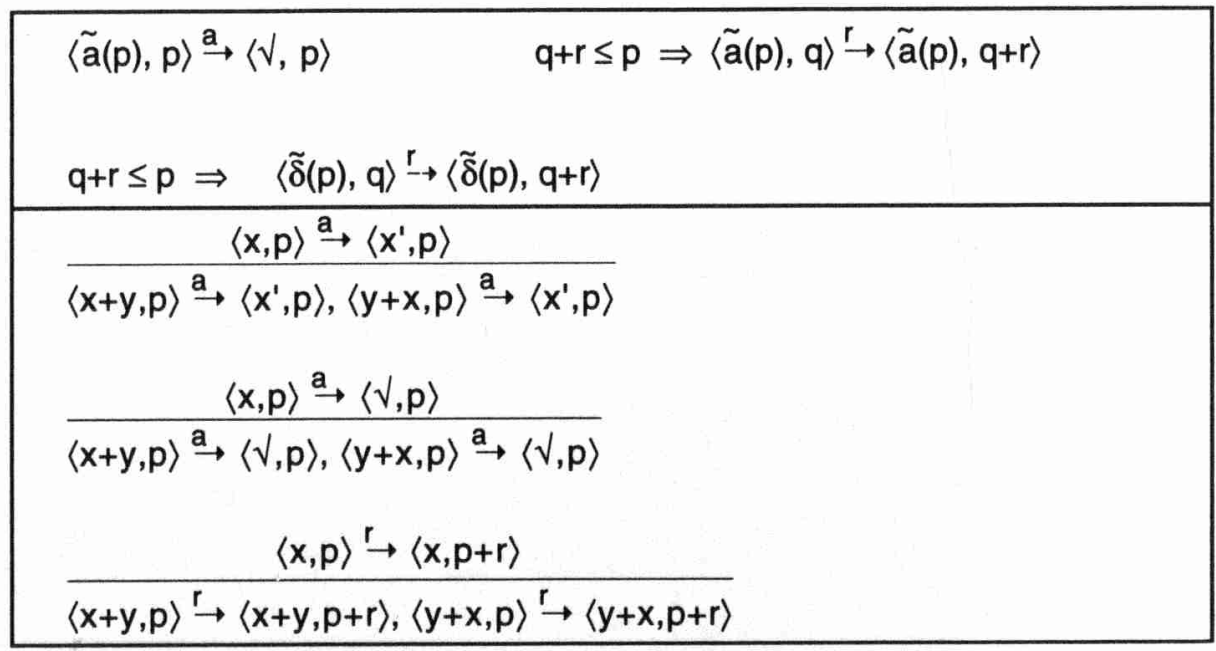




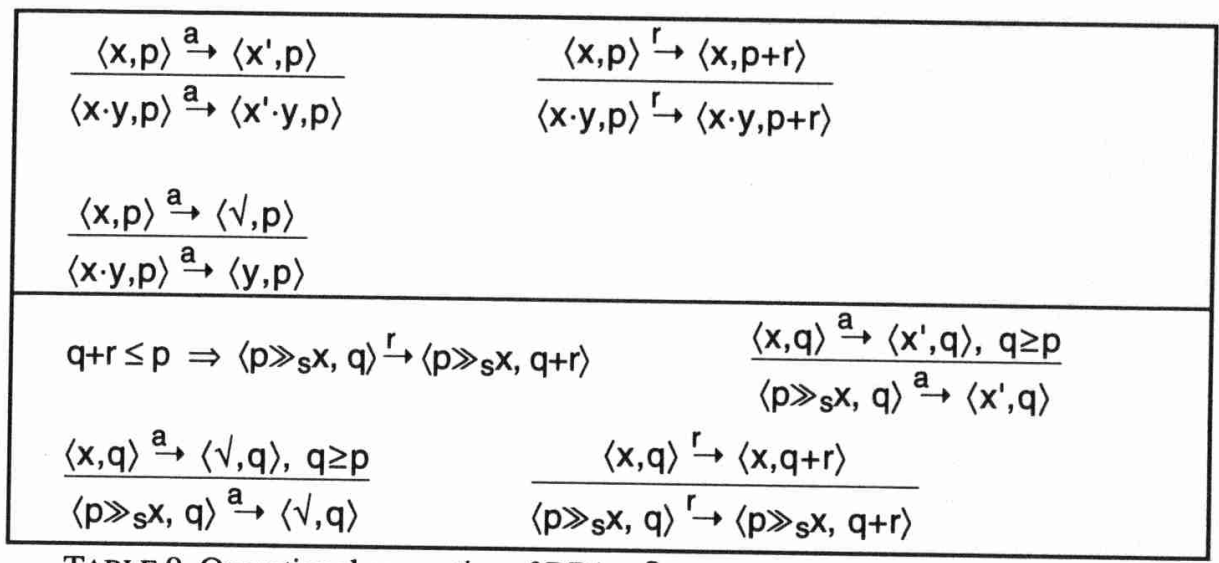

TABLE 9. Operational semantics of BPAsp $\delta$.

\subsection{Parallel composition.}

In a setting with urgent actions, it makes sense to define parallel composition without communication, as urgent actions composed in parallel will show interleaving. Thus, $a(2) \| b(2)$ forces synchronisation, but we have $\tilde{a}(2) \| \tilde{b}(2)=\tilde{a}(2) \cdot \tilde{b}(2)+\tilde{b}(2) \cdot \tilde{a}(2)$. In table $10, \mathrm{H} \subseteq \mathrm{A}, \mathrm{a}, \mathrm{b} \in \mathrm{A}_{\delta}$. SOS rules are in table 11 .

$p \leq q \Rightarrow \tilde{a}(p) \oplus \tilde{b}(q)=\tilde{a}(p)$

$p>q \Rightarrow \tilde{a}(p) \oplus \tilde{b}(q)=\tilde{\delta}(q)$

$\mathrm{X} \oplus \mathrm{Y} \cdot \mathrm{Z}=\mathrm{X} \oplus \mathrm{Y}$

$X \oplus(Y+Z)=(X \oplus Y)+(X \oplus Z)$

$X \cdot Y \oplus Z=(X \oplus Y) \cdot Z$

$(X+Y) \oplus Z=(X \oplus Z)+(Y \oplus Z)$

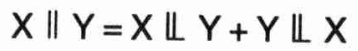

$X=0 \gg_{s} Z \Rightarrow \tilde{a}(p) \Perp X=(\tilde{a}(p) \oplus X) \cdot X$

$Y=0 \gg_{s} Z \Rightarrow \tilde{a}(p) \cdot X \Perp Y=(\tilde{a}(p) \oplus Y) \cdot(X \| Y)$

TABLE 10. Additional axioms of PAsp $\delta$. 
178

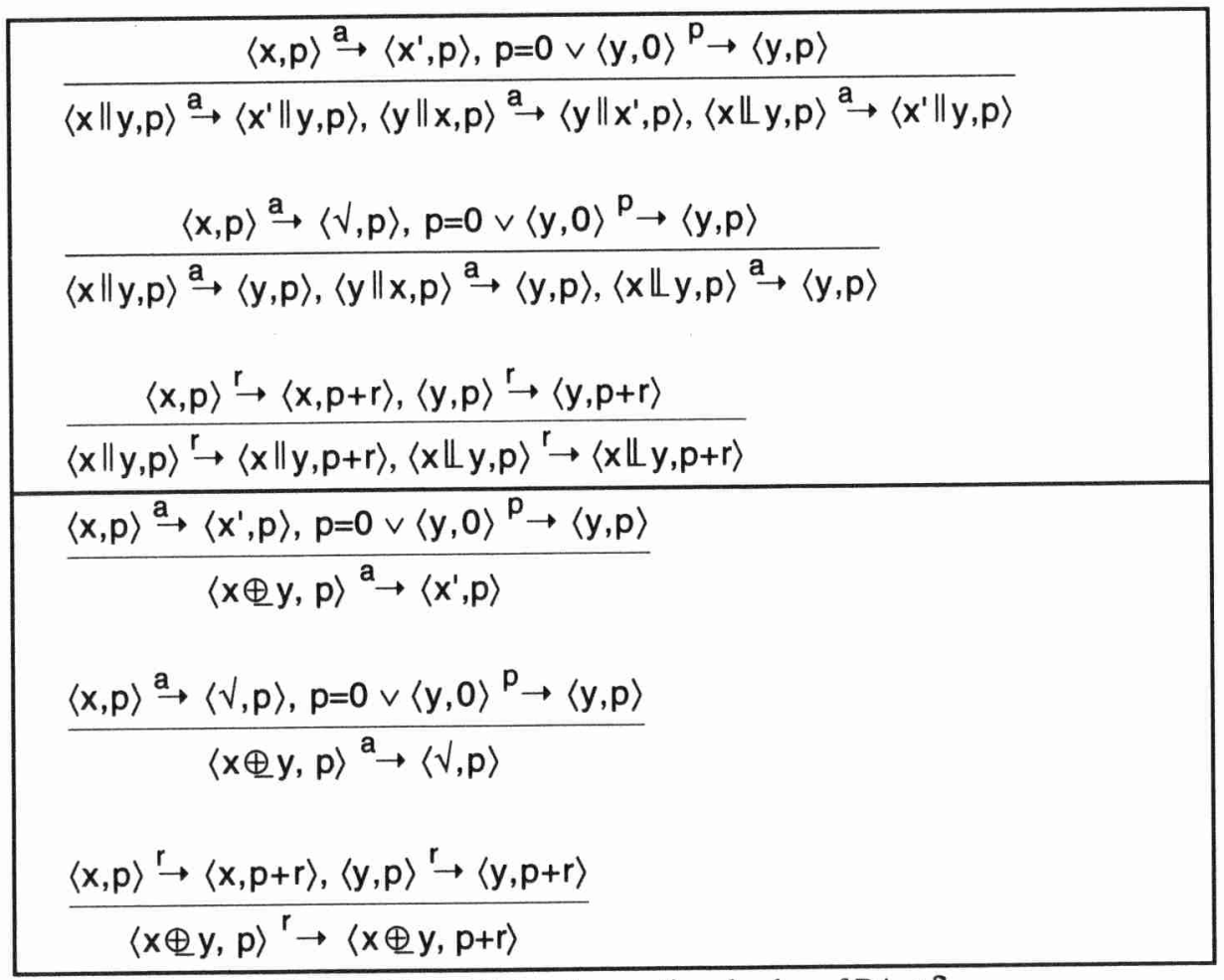

TABLE 11. Additional operational rules of PAs $\rho$.

6.4 Communication.

Additional axioms of $A C P s \rho$ are in table 12. In table $12, H \subseteq A, a, b \in A_{\delta}$. 


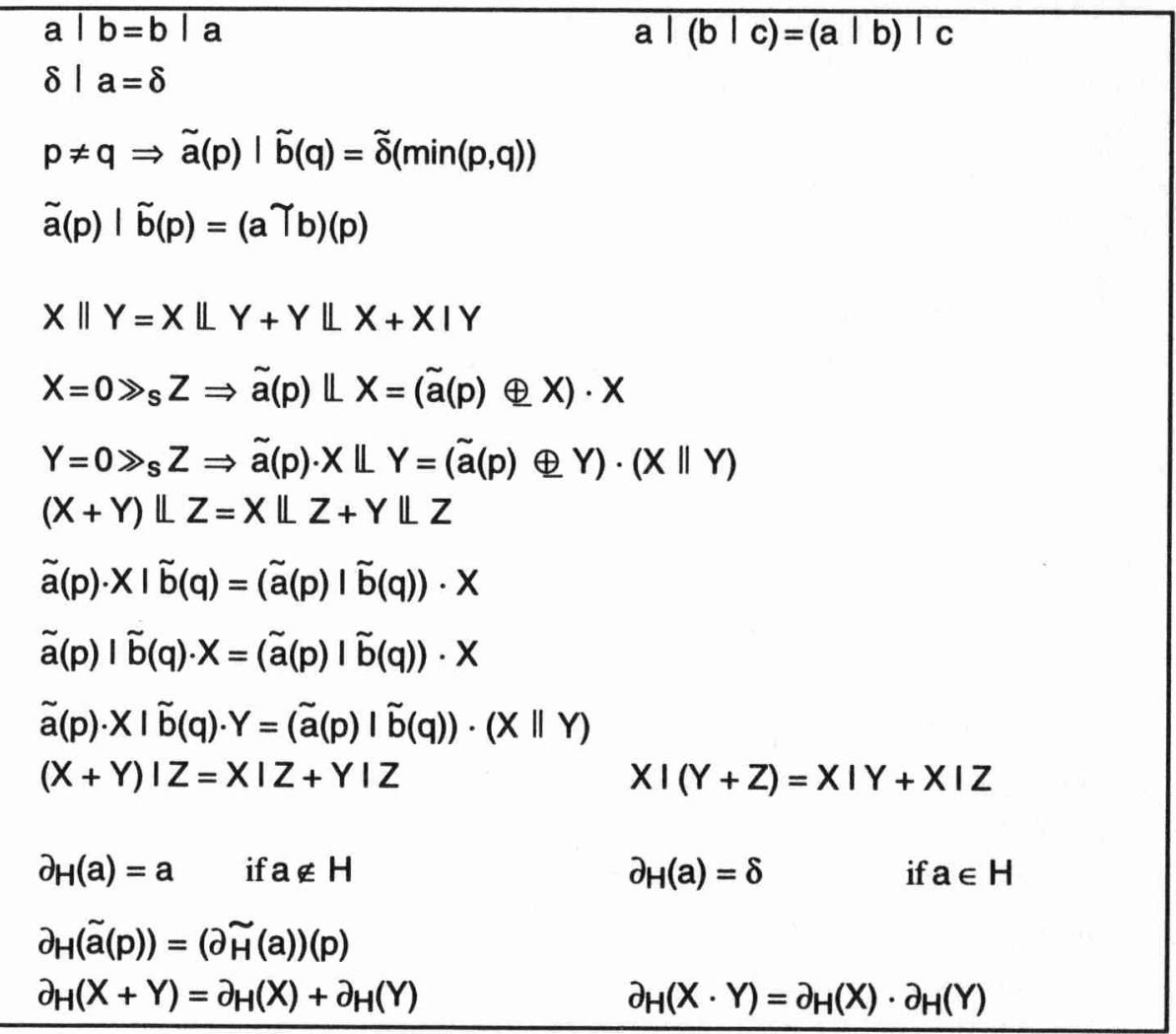

TABLE 12. Additional axioms of ACPsp.

$\langle x, p\rangle \stackrel{a}{\rightarrow}\left\langle x^{\prime}, p\right\rangle,\langle y, p\rangle \stackrel{b}{\rightarrow}\left\langle y^{\prime}, p\right\rangle, a \mid b=c \neq \delta$

$\langle x \| y, p\rangle \stackrel{c}{\rightarrow}\left\langle x^{\prime} \| y^{\prime}, p\right\rangle,\langle x \mid y, p\rangle \stackrel{c}{\rightarrow}\left\langle x^{\prime} \| y^{\prime}, p\right\rangle$

$\langle x, p\rangle \stackrel{a}{\rightarrow}\left\langle x^{\prime}, p\right\rangle,\langle y, p\rangle \stackrel{b}{\rightarrow}\langle\sqrt{ }, p\rangle, a \mid b=c \neq \delta$

$\langle x \| y, p\rangle \stackrel{c}{\rightarrow}\left\langle x^{\prime}, p\right\rangle,\langle y \| x, p\rangle^{c} \rightarrow\left\langle x^{\prime}, p\right\rangle,\langle x \mid y, p\rangle \stackrel{c}{\rightarrow}\left\langle x^{\prime}, p\right\rangle,\langle y \mid x, p\rangle \stackrel{c}{\rightarrow}\left\langle x^{\prime}, p\right\rangle$

$\langle x, p\rangle \stackrel{a}{\rightarrow}\langle\sqrt{ }, p\rangle,\langle y, p\rangle \stackrel{b}{\rightarrow}\langle\sqrt{ }, p\rangle, a \mid b=c \neq \delta$

$\langle x \| y, p\rangle \stackrel{c}{\rightarrow}\langle\sqrt{ }, p\rangle,\langle x \mid y, p\rangle \stackrel{c}{\rightarrow}\langle\sqrt{ }, p\rangle$

$\frac{\langle x, p\rangle \stackrel{r}{\rightarrow}\langle x, p+r\rangle,\langle y, p\rangle \stackrel{r}{\rightarrow}\langle y, p+r\rangle}{\langle x \mid y, p\rangle \stackrel{r}{\rightarrow}\langle x \mid y, p+r\rangle}$ 


$$
\begin{aligned}
& \frac{\langle x, p\rangle \stackrel{a}{\rightarrow}\left\langle x^{\prime}, p\right\rangle, a \notin H}{\left\langle\partial_{H}(x), p\right\rangle \stackrel{a}{\rightarrow}\left\langle\partial_{H}\left(x^{\prime}\right), p\right\rangle} \quad \frac{\langle x, p\rangle \stackrel{r}{\rightarrow}\langle x, p+r\rangle}{\left\langle\partial_{H}(x), p\right)^{r} \rightarrow\left\langle\partial_{H}(x), p+r\right\rangle} \\
& \frac{\langle x, p\rangle \stackrel{a}{\rightarrow}\langle\sqrt{ }, p\rangle, a \notin H}{\left\langle\partial_{H}(x), p\right\rangle \stackrel{a}{\rightarrow}\langle\sqrt{ }, p\rangle}
\end{aligned}
$$

TABLE 13. Additional operational rules of ACPs $\rho$.

\subsection{Initial abstraction.}

We extend with initial abstraction as in 2.4, and obtain ACPs $\rho \sqrt{ }$. We extend the semantics as in 2.12 .

$$
\begin{aligned}
& \tilde{a}[p]=V_{s} q \cdot \tilde{a}(p+q) \\
& V_{S} p . F=V_{S} q . F[q / p] \quad \text { if } q \text { is not free in } F \\
& p \gg_{s} V_{s} q \cdot F=p \gg_{s} F[p / q] \\
& V_{s} q . V_{s} p . F=V_{s} q . F[q / p] \\
& X=V_{s} p . X \\
& \forall p \in \mathbb{R}_{\geq 0} p \gg_{s} X=p \gg_{s} Y \Rightarrow X=Y \\
& \left(V_{s} p \cdot F\right)+X=V_{s} p \cdot\left(F+p \gg_{s} X\right) \quad\left(V_{s} p \cdot F\right) \cdot X=V_{s} p \cdot(F \cdot X)
\end{aligned}
$$

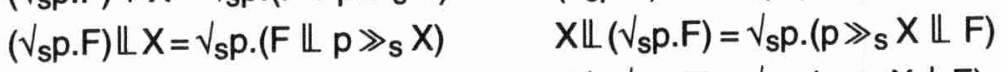

$$
\begin{aligned}
& \left(V_{s} p . F\right)\left|X=V_{s} p .\left(F \mid p \gg_{s} X\right) \quad X\right|\left(V_{s} p . F\right)=V_{s} p .\left(p \gg_{s} X \mid F\right) \\
& \partial_{H}\left(V_{s} p . F\right)=V_{s} p . \partial_{H}(F) \quad\left(V_{s} p . F\right) \oplus X=V_{s} p .\left(F \oplus p \gg_{S} X\right) \\
& X \oplus\left(V_{s} p . F\right)=V_{s} p \cdot\left(p \gg_{S} X \oplus F\right)
\end{aligned}
$$

TABLE 14. Axioms for initial abstraction.

\section{Standard real time process algebra: relative time.}

\subsection{Basic process algebra.}

The signature of BPA $_{\text {st }}$ is as follows:
- constants $\tilde{a}$
urgent $a(a \in A)$.
- functions $+: \mathrm{P} \times \mathrm{P} \rightarrow \mathrm{P}$ alternative composition

$$
\therefore \mathrm{P} \times \mathrm{P} \rightarrow \mathrm{P}
$$ sequential composition$$
\sigma_{\approx}^{r}: P \rightarrow P
$$ positive time shift $(r 0)$.

In table $15, r, p>0$. 


\begin{tabular}{|ll|}
\hline$X+Y=Y+X$ & $\sigma_{\approx}^{r} \circ \sigma \stackrel{p}{\approx}(X)=\sigma_{\approx}^{r+p}(X)$ \\
$(X+Y)+Z=X+(Y+Z)$ & $\sigma_{\approx}^{r}(X)+\sigma_{\approx}^{r}(Y)=\sigma_{\approx}^{r}(X+Y)$ \\
$X+X=X$ & $\sigma_{\approx}^{r}(X) \cdot Y=\sigma_{\approx}^{r}(X \cdot Y)$ \\
$(X+Y) \cdot Z=X \cdot Z+Y \cdot Z$ & \\
$(X \cdot Y) \cdot Z=X \cdot(Y \cdot Z)$ & \\
\hline
\end{tabular}

TABLE 15. BPA st.

The operational semantics is defined by action rules with two types of transitions:

$$
\begin{aligned}
& \text { idle } \subseteq P \times \mathbb{R}_{>0} \times P, \text { notation } x \stackrel{r}{\rightarrow} x^{\prime} \\
& \text { step } \subseteq P \times A \times P, \text { notation } x \stackrel{a}{\rightarrow} x^{\prime} .
\end{aligned}
$$

Here $r>0, a \in A$.

$$
\begin{aligned}
& \underset{\tilde{a}}{\stackrel{a}{\rightarrow} \sqrt{ } \quad \frac{x^{a} \rightarrow x^{\prime}}{x \cdot y^{a} \rightarrow x^{\prime} \cdot y} \quad \frac{x^{a} \rightarrow \sqrt{ }}{x \cdot y^{a} \rightarrow y}} \\
& \frac{x^{a} \rightarrow x^{\prime}}{x+y^{a} \rightarrow x^{\prime}, y+x^{a} \rightarrow x^{\prime}} \\
& \frac{x^{a} \rightarrow \sqrt{ }}{x+y^{a} \rightarrow \sqrt{ }, y+x^{a} \rightarrow \sqrt{ }} \\
& \sigma_{\approx}^{r}(x) \stackrel{r}{\rightarrow} x \\
& p, r>0 \Rightarrow \sigma \stackrel{\sim}{p+r}(x) \stackrel{r}{\rightarrow} \underset{\approx}{\stackrel{p}{p}}(x) \\
& \frac{x^{r} \rightarrow x^{\prime}}{\sigma \stackrel{p}{\sim}(x) \stackrel{p+r}{\rightarrow} x^{\prime}} \\
& \frac{x^{r} \rightarrow x^{\prime}}{x \cdot y^{r} \rightarrow x^{\prime} \cdot y} \\
& \frac{x^{r} \rightarrow x^{\prime}, y^{r} \rightarrow y^{\prime}}{x+y^{r} \rightarrow x^{\prime}+y^{\prime}} \\
& \frac{x^{r} \rightarrow x^{\prime}, y^{r} \rightarrow}{x+y^{r} \rightarrow x^{\prime}, y+x^{r} \rightarrow x^{\prime}}
\end{aligned}
$$

TABLE 16. Operational semantics of $\mathrm{BPA}_{\text {st. }}$.

\subsection{Inaction.}

$\mathrm{BPA}_{\delta \mathrm{st}}$ is obtained by introducing $\widetilde{\delta}$ as a constant representing inaction. The axioms for $\widetilde{\delta}$ are standard (table 17).

The operational meaning of $\widetilde{\delta}$ is a process that allows no step whatsoever. In the graph model, $\widetilde{\tilde{\delta}}$ will be modeled by the tree with no edges, and one node which is the root but not a termination node. 
$\mathrm{X}+\widetilde{\delta}=\mathrm{X} \quad \tilde{\delta} \cdot \mathrm{X}=\widetilde{\delta}$

TABLE 17. Additional axioms of $B P A \delta s t$.

7.3 Parallel composition.

Axioms are in table $18\left(a \in A_{\delta}, x, y, z \in P, r, p>0\right)$.

$$
\begin{aligned}
& \widetilde{\tilde{a}} \oplus \widetilde{\mathrm{b}}=\tilde{\mathrm{a}} \\
& \tilde{\mathrm{a}} \oplus \sigma_{\approx}^{r}(X)=\tilde{\mathrm{a}} \\
& \sigma_{\approx}^{r}(X) \oplus \widetilde{\tilde{a}}=\widetilde{\delta} \\
& \sigma_{\approx}^{r}(X) \oplus \sigma_{\approx}^{r}(Y)=\sigma_{\approx}^{r}(X \oplus Y) \\
& X \oplus Y \cdot Z=X \oplus Y \\
& X \oplus(Y+Z)=(X \oplus Y)+(X \oplus Z) \\
& X \cdot Y \oplus Z=(X \oplus Y) \cdot Z \\
& (X+Y) \oplus Z=(X \oplus Z)+(Y \oplus Z) \\
& r \gg_{s t} \tilde{\tilde{a}}=\sigma_{\approx}^{r}(\widetilde{\tilde{\delta}}) \\
& r \gg_{s t}(X+Y)=r \gg_{s t} X+r \gg_{s t} Y \\
& r \gg_{s t}(X \cdot Y)=\left(r \gg_{s t} X\right) \cdot Y \\
& r \gg_{s t} \sigma_{\approx}^{r}(X)=\sigma_{\approx}^{r}(X) \\
& p>r \Rightarrow p \gg_{s t} \sigma_{\approx}^{r}(X)=\sigma_{\approx}^{r}\left((p-r) \gg_{s t} X\right)
\end{aligned}
$$

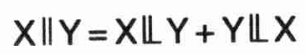

$$
\begin{aligned}
& \tilde{\tilde{a}} \Perp x=\tilde{a} \cdot x \\
& (\tilde{\mathrm{a}} \cdot X) \| Y=\tilde{\tilde{a}} \cdot(X \| Y) \\
& (X+Y) \amalg Z=X \Perp Z+Y \amalg Z \\
& \sigma_{\approx}^{r}(X) \amalg Y=\left(\sigma_{\approx}^{r}(X) \oplus Y\right) \mathbb{L}\left(r \gg_{s t} Y\right)
\end{aligned}
$$

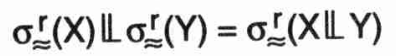


183

$$
\begin{aligned}
& \frac{x^{a} \rightarrow x^{\prime}}{x\left\|y \stackrel{a}{\rightarrow} x^{\prime}\right\| y, y\left\|x^{a} \rightarrow y\right\| x^{\prime}, x\left\|y^{a} \rightarrow x^{\prime}\right\| y} \\
& \frac{x^{a} \rightarrow \sqrt{x} \stackrel{a}{x} y, y\left\|x^{a} \rightarrow y, x\right\| y^{a} \rightarrow y}{x\left\|y^{r} \rightarrow x^{\prime}\right\| y^{\prime}, x\left\|y^{r} \rightarrow x^{\prime}\right\| y^{\prime}} \\
& \frac{x^{r} \rightarrow x^{\prime}, r \geq p}{p \gg_{s t} x^{r} \rightarrow x^{\prime}} \\
& \frac{x^{r} \rightarrow x^{\prime}, r<p}{p \gg_{s t} x^{r} \rightarrow(p-r) \gg_{s t} x} \\
& \frac{x \stackrel{a}{\rightarrow} x^{\prime}}{x \oplus y \stackrel{a}{\rightarrow} x^{\prime}} \quad \frac{x \stackrel{a}{\rightarrow} \sqrt{ }}{x \oplus y^{a} \stackrel{\sqrt{ }}{\rightarrow} \sqrt{ }} \\
& \frac{\underset{\mathrm{r}}{\rightarrow} \mathrm{x}^{\prime}, \mathrm{y}^{\mathrm{r}} \rightarrow \mathrm{y}^{\prime}}{x \oplus \mathrm{r}^{\mathrm{r}} \rightarrow \mathrm{x}^{\prime} \oplus \mathrm{y}^{\prime}}
\end{aligned}
$$

TABLE 19. Additional operational rules of $\mathrm{PA}_{\delta s t}$.

7.4 Communication.

The axioms of $\mathrm{ACP}_{\text {st }}$ are in table 20. In table $20, \mathrm{H} \subseteq \mathrm{A}, \mathrm{r}>0, \mathrm{a}, \mathrm{b} \in \mathrm{A}_{\delta}$. 


$$
\begin{aligned}
& \widetilde{a} \mid \widetilde{b}=a{ }^{b} b \\
& (\tilde{\mathrm{a}} \cdot \mathrm{X}) \mid \tilde{\mathrm{b}}=(\tilde{\mathrm{a}} \mid \tilde{\mathrm{b}}) \cdot \mathrm{X}
\end{aligned}
$$

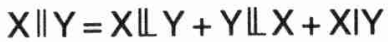

$$
\begin{aligned}
& \tilde{\tilde{a}} \mid(\tilde{\mathrm{b}} \cdot X)=(\tilde{\tilde{a}} \mid \widetilde{b}) \cdot X \\
& \tilde{\tilde{a}} \| X=\tilde{a} \cdot X \\
& (\tilde{\mathrm{a}} \cdot X) \mid(\tilde{\mathrm{b}} \cdot Y)=(\tilde{\mathrm{a}} \mid \tilde{\mathrm{b}}) \cdot(X \| Y) \\
& (\tilde{\mathrm{a}} \cdot X) \| Y=\tilde{\tilde{a}} \cdot(X \| Y) \\
& (X+Y) \mid Z=X I Z+Y I Z
\end{aligned}
$$

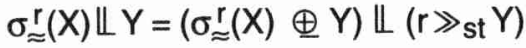

$$
\begin{aligned}
& X I(Y+Z)=X I Y+X I Z
\end{aligned}
$$

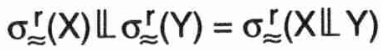

$$
\begin{aligned}
& \widetilde{\tilde{a}} \mid \sigma_{\approx}^{r}(X)=\widetilde{\delta}
\end{aligned}
$$

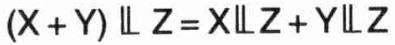

$$
\begin{aligned}
& \sigma_{\approx}^{r}(X) \mid \approx \tilde{a}=\widetilde{\delta} \\
& (\widetilde{\tilde{a}} \cdot \mathrm{X}) \mid \sigma_{\approx}^{\mathrm{r}}(\mathrm{Y})=\widetilde{\delta} \\
& \partial_{\mathrm{H}}(\widetilde{\tilde{a}})=\widetilde{\tilde{a}} \quad \text { if } \mathrm{a} \notin \mathrm{H} \\
& \sigma_{\approx}^{r}(X) \mid(\tilde{\tilde{a}} \cdot Y)=\widetilde{\delta} \\
& \partial_{\mathrm{H}}(\widetilde{\tilde{a}})=\widetilde{\delta} \quad \text { if } \mathrm{a} \in \mathrm{H} \\
& \sigma_{\approx}^{r}(X) \mid \sigma_{\approx}^{r}(Y)=\sigma_{\approx}^{r}(X \mid Y) \\
& \partial_{H}(X+Y)=\partial_{H}(X)+\partial_{H}(Y) \\
& \partial_{H}(X \cdot Y)=\partial_{H}(X) \cdot \partial_{H}(Y) \\
& \partial_{H}\left(\sigma_{\approx}^{r}(X)\right)=\sigma_{\approx}^{r}\left(\partial_{H}(X)\right)
\end{aligned}
$$

TABLE 20. Additional axioms of $\mathrm{ACP}_{\text {st }}$.

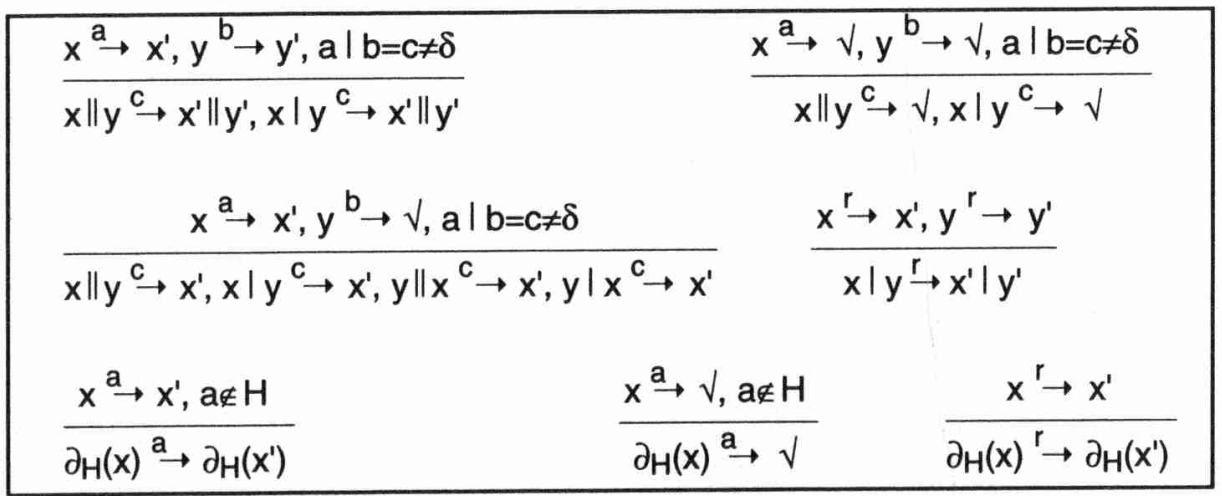

TABLE 21. Additional operational rules of $\mathrm{ACP}_{\text {st. }}$.

\subsection{Remark.}

Consider the axiom system $\mathrm{ACP}_{s t}$, and omit all signature elements refering to timing, i.e. omit the operators $\sigma_{\approx}^{r}, r \gg_{s t}, \gg_{s}, \oplus$. The remaining signature is exactly the signature of the untimed theory ACP of $[5,6]$ (interpreting $\widetilde{a}$ as a, $\widetilde{\delta}$ as $\delta$ ), and the axioms over this signature are exactly the axioms of ACP. Thus, the untimed theory 
$\mathrm{ACP}$ is obtained from $\mathrm{ACP}_{\text {st }}$ by throwing away all timing information, or, put another way, $\mathrm{ACP}_{\text {st }}$ over the one-point time domain $\mathcal{T}=\{0\}$ gives the untimed theory ACP.

\section{Translations.}

This section is based on [9]. We provide interpretations of the timing constructs of other process algebras, involving urgent actions, into our real time process algebra. [.] denotes the interpretation function. More information, involving the treatment of parallel composition and the modeling of maximal progress, can be found in [9].

$$
\begin{aligned}
& \text { 8.1 ATP [13]. } \\
& \text { [0] }=\quad \widetilde{\delta} \\
& {[\delta]=\quad \tilde{\delta}[\infty]=\int_{p \geq 0} \tilde{\delta}[p]} \\
& {[\dot{\mathrm{a}} \mathrm{P}]=\quad \tilde{\mathrm{a}} \cdot[\mathrm{P}]} \\
& {[\tilde{a} P]=\quad \int_{p \geq 0} \tilde{a}[p] \cdot[P]} \\
& \llbracket P \oplus \mathrm{Q} \rrbracket=\quad([\mathrm{P}] \oplus[\mathrm{Q}])+([\mathrm{Q} \rrbracket \oplus[\mathrm{P}]) \quad \text { (strong choice) } \\
& {[P \stackrel{d}{\triangleleft} Q]=\quad([P \rrbracket \oplus \tilde{\delta}[d])+(d \gg s t[Q]) .}
\end{aligned}
$$

\subsection{TCCS of Wang Yi [16].}

$$
\begin{array}{ll}
{[N I L]=} & \tilde{\delta}[\infty]=\int_{p \geq 0} \tilde{\delta}[p] \\
{[\tau]=} & \tilde{\tau} \\
{[a . P]=} & \int_{p \geq 0} \tilde{a}[p] \cdot[P] \quad(a \neq \tau) \\
{[\varepsilon(r) \cdot P]=} & \sigma_{\approx}^{r}([P]) . \\
{[P+Q]=} & ([P] \oplus[Q])+([Q] \oplus[P]) \quad \text { (strong choice). }
\end{array}
$$

Note that [16] uses a maximal progress assumption. In order to model this, we need to use a priority operator, as in [3]. This has been worked out in [9].

\subsection{TCCS of Moller \& Tofts $[11,12]$. $[0]=\quad \tilde{\delta}$}




$$
\begin{array}{lll}
{[0]=} & \tilde{\delta}[\infty]=\int_{p \geq 0} \tilde{\delta}[p] & \text { (only in [12]) } \\
{[\text { a.P }]=} & \tilde{a} \cdot[P] & \text { (only in [12]) } \\
{[\text { a.P }]=} & \int_{p \geq 0} \tilde{a}[p] \cdot[P] & \\
{[(r) . P]=} & \sigma_{\approx}^{r}([P]) & \text { (only in }[11]) \\
{[\delta . P]=} & \int_{r \geq 0} \sigma \approx[(P] \oplus \widetilde{\delta}) & \text { (strong choice; denoted }+ \text { in }[11]) \\
{[P+Q]=} & ([P] \oplus[Q])+([Q] \oplus[P]) & \text { (weak choice; denoted } \oplus \text { in }[11]) . \\
{[P+Q]=} & {[P]+[Q]} &
\end{array}
$$

8.4 Chen [7].

$$
\begin{array}{ll}
{[N I L]=} & \tilde{\delta}[\infty]=\int_{p \geq 0} \tilde{\delta}[p] \\
{\left[\left.a(p)\right|_{e} ^{b} \cdot P\right]=} & \int_{p \in[b, e]} \tilde{a}[p] \cdot[P]+\tilde{\delta}[e] \\
{[P+Q]=} & {[P]+[Q]} \\
{[(r) \cdot P]=} & \sigma_{\approx}^{r}([P]) .
\end{array}
$$

\section{Conclusion.}

We have modeled urgent actions in our real time process algebra, by extending the time domain to include nonstandard real numbers, and interpreting urgent actions as an alternative composition of normal timed actions of ACPp. In this way, process calculi involving urgent actions can be interpreted in ACP. Also, we have indicated subalgebras that allow an operational semantics where actions and time are separated.

We conclude that our real time process algebra provides a general framework, in which many features occurring in the literature can be modeled.

\section{References.}

1. J.C.M. Baeten \& J.A. Bergstra. Real time process algebra. Formal Aspects of Computing 1991; 3:142-188. 
2. J.C.M. Baeten \& J.A. Bergstra. Discrete time process algebra. In: W.R. Cleaveland (ed.), Proc. CONCUR'92, Stony Brook. Springer Verlag 1992, pp. 401420 (Lecture Notes in Computer Science no. 630).

3. J.C.M. Baeten \& J.A. Bergstra. Real space process algebra. Formal Aspects of Computing 1993; 5:481-529.

4. J.C.M. Baeten, J.A. Bergstra \& J.W. Klop. Syntax and defining equations for an interrupt mechanism in process algebra. Fund. Inf. 1986; IX:127-168.

5. J.C.M. Baeten \& W.P. Weijland. Process algebra. Cambridge Tracts in Theor. Comp. Sci. 18, Cambridge University Press 1990.

6. J.A. Bergstra \& J.W. Klop. Process algebra for synchronous communication. Information \& Control 1984; 60:109-137.

7. L. Chen. Timed processes: models, axioms and decidability. Ph.D. thesis, University of Edinburgh 1993.

8. W.J. Fokkink \& A.S. Klusener. Real time process algebra with prefixed integration. Report CS-R9219, CWI, Amsterdam 1992.

9. A.S. Klusener. Models and axioms for a fragment of real time process algebra. Ph.D. Thesis, Eindhoven University of Technology 1993.

10. W.A.J. Luxemburg. Non-standard analysis (lectures on A. Robinson's theory of infinitesimals and infinitely large numbers). California Institute of Technology, Pasadena 1962.

11. F. Moller \& C. Tofts. A temporal calculus of communicating systems. In: J.C.M. Baeten \& J.W. Klop (eds.), Proc. CONCUR'90, Amsterdam. Springer Verlag 1990, pp. 401-415 (Lecture Notes in Computer Science no. 458).

12. F. Moller \& C. Tofts. Behavioural abstraction in TCCS. In: W. Kuich (ed.), Proc. ICALP 92, Vienna. Springer Verlag 1992 (Lecture Notes in Computer Science 623).

13. X. Nicollin \& J. Sifakis. The algebra of timed processes ATP: theory and application (revised version). Report RT-C26, IMAG Grenoble 1991.

14. G.D. Plotkin. A structural approach to operational semantics. Report DAIMI FN19, Comp. Sci. Dept., Aarhus University 1981.

15. A. Robinson. Non-standard analysis. Studies in Logic and the Foundations of Mathematics, North-Holland, Amsterdam 1966.

16. Wang Yi. Real-time behaviour of asynchronous agents. In: J.C.M. Baeten \& J.W. Klop (eds.), Proc. CONCUR'90, Amsterdam, Springer Verlag 1990, pp. 502-520 (Lecture Notes in Computer Science 458). 